\title{
Hausdorff dimension and conformal dynamics II: Geometrically finite rational maps
}

\author{
Curtis T. McMullen
}

\section{Contents}

1. Introduction .......................................... 536

2. The basic invariants................................. 540

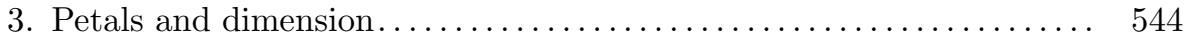

4. Poincaré series ...................................... 546

5. Dynamics on the radial Julia set ...................... 550

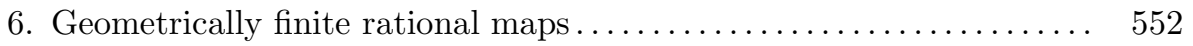

7. Creation of parabolics ............................... 556

8. Linearizing parabolic dynamics .......................... 560

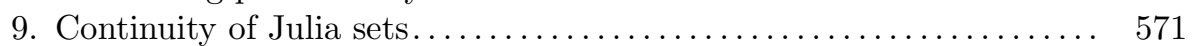

10. Parabolic and Poincaré series............................ 575

11. Continuity of Hausdorff dimension....................... 578

12. Julia sets of dimension near two ........................ 582

13. Quadratic polynomials ............................. 587

14. Examples of discontinuity ........................... 589

Research partially supported by the NSF. 
Abstract. This paper investigates several dynamically defined dimensions for rational maps $f$ on the Riemann sphere, providing a systematic treatment modeled on the theory for Kleinian groups.

We begin by defining the radial Julia set $J_{\text {rad }}(f)$, and showing that every rational map satisfies

$$
\text { H. } \operatorname{dim} J_{\text {rad }}(f)=\alpha(f)
$$

where $\alpha(f)$ is the minimal dimension of an $f$-invariant conformal density on the sphere.

A rational map $f$ is geometrically finite if every critical point in the Julia set is preperiodic. In this case we show

$$
\text { H. } \operatorname{dim} J_{\mathrm{rad}}(f)=\mathrm{H} \cdot \operatorname{dim} J(f)=\delta(f),
$$

where $\delta(f)$ is the critical exponent of the Poincaré series; and $f$ admits a unique normalized invariant density $\mu$ of dimension $\delta(f)$.

Now let $f$ be geometrically finite and suppose $f_{n} \rightarrow f$ algebraically, preserving critical relations. When the convergence is horocyclic for each parabolic point of $f$, we show $f_{n}$ is geometrically finite for $n \gg 0$ and $J\left(f_{n}\right) \rightarrow J(f)$ in the Hausdorff topology. If the convergence is radial, then in addition we show $\mathrm{H} . \operatorname{dim} J\left(f_{n}\right) \rightarrow \mathrm{H} . \operatorname{dim} J(f)$.

We give examples of horocyclic but not radial convergence where H. $\operatorname{dim} J\left(f_{n}\right) \rightarrow 1>$ H. $\operatorname{dim} J(f)=1 / 2+\epsilon$. We also give a simple demonstration of Shishikura's result that there exist $f_{n}(z)=z^{2}+c_{n}$ with H. $\operatorname{dim} J\left(f_{n}\right) \rightarrow 2$.

The proofs employ a new method that reduces the study of parabolic points to the case of elementary Kleinian groups.

Mathematics Subject Classification (2000). Primary 58F23; Secondary 58F11, 30F40.

Keywords. Complex dynamics, iterated rational maps, Julia sets, Hausdorff dimension.

\section{Introduction}

Let $f: \widehat{\mathbb{C}} \rightarrow \widehat{\mathbb{C}}$ be a rational map on the Riemann sphere, of degree $d \geq 2$. In this paper we study the equality of several dynamically defined dimensions for $f$, and their variation as a function of $f$.

To pattern the theory after that of Kleinian groups, we define the radial Julia set of a rational map and a notion of geometric finiteness in the dynamical setting. As a bridge between the two subjects, we also develop a new technique that reduces the study of parabolic bifurcations of rational maps to the case of Möbius transformations.

To summarize the main results, we first introduce various dimensions determined by the dynamics of a rational map $f$. Distances and derivatives are measured in the spherical metric.

1. The Julia set $J(f)$ is the closure of the repelling periodic points for $f$. Our first invariant is its Hausdorff dimension, H. $\operatorname{dim} J(f)$.

2. We can also consider the dimension of the radial Julia set $J_{\text {rad }}(f)$, consisting of those $z$ for which arbitrarily small neighborhoods of $z$ can be expanded 
univalently by the dynamics to balls of definite size.

3. A compact set $X \subset \widehat{\mathbb{C}}$ is expanding for $f$ if $f(X) \subset X$ and $f$ uniformly expands a smooth metric defined near $X$. The hyperbolic dimension, hyp-dim $(f)$ is the supremum of the Hausdorff dimensions of such expanding sets $X$.

4. An $f$-invariant density of dimension $\alpha>0$ is a finite positive measure $\mu$ on $\widehat{\mathbb{C}}$ such that

$$
\mu(f(E))=\int_{E}\left|f^{\prime}(x)\right|^{\alpha} d \mu
$$

whenever $f \mid E$ is injective. The critical dimension $\alpha(f)$ is the minimum possible dimension of an $f$-invariant density.

5. The Poincaré series is defined by

$$
P_{s}(f, x)=\sum_{f^{n}(y)=x}\left|\left(f^{n}\right)^{\prime}(y)\right|^{-s},
$$

and the supremum of those $s \geq 0$ such that $P_{s}(f, x)=\infty$ for all $x \in \widehat{\mathbb{C}}$ is the critical exponent $\delta(f)$.

In general one knows (§2):

Theorem 1.1. For any rational map $f$,

$$
\alpha(f)=\operatorname{hyp}-\operatorname{dim}(f)=\text { H. } \operatorname{dim} J_{\text {rad }}(f) .
$$

For more complete results, we introduce some restrictions on $f$. A rational map $f$ is expanding if its Julia set contains no critical points or parabolic points. More generally, $f$ is geometrically finite if every critical point in $J(f)$ has a finite forward orbit. Geometrically finite maps can have attracting, superattracting and parabolic basins, but no Siegel disks or Herman rings. In $\S 6$ we show:

Theorem 1.2. Let $f$ be geometrically finite. Then

$$
\delta(f)=\text { H. } \operatorname{dim} J_{\mathrm{rad}}(f)=\text { H. } \operatorname{dim} J(f),
$$

and the Poincaré series $P_{s}(f, x)$ diverges at $s=\delta(f)$ for all $x \in \widehat{\mathbb{C}}$.

Moreover the sphere admits a unique normalized $f$-invariant density $\mu$ of $d i$ mension $\delta(f)$. The canonical density $\mu$ is nonatomic and supported on $J_{\mathrm{rad}}(f)$, and any $f$-invariant density on the Julia set is either purely atomic or proportional to $\mu$. 
Next we discuss the behavior of the Julia set and its dimension under limits of rational maps. We say $f_{n} \rightarrow f$ algebraically if $\operatorname{deg} f_{n}=\operatorname{deg} f$ and the coefficients of $f_{n}$ (as a ratio of polynomials) can be chosen to converge to those of $f$. When $f$ is expanding, algebraic convergence suffices to guarantee $J\left(f_{n}\right) \rightarrow J(f)$ and H. $\operatorname{dim} J\left(f_{n}\right) \rightarrow$ H. $\operatorname{dim} J(f)$. When $f$ is geometrically finite, however, one must also control parabolic bifurcations and critical points to achieve continuity.

To describe the condition on parabolic points, suppose $\lambda_{n}=\exp \left(L_{n}+i \theta_{n}\right) \rightarrow 1$ in $\mathbb{C}^{*}$. We say $\lambda_{n} \rightarrow 1$ radially if

$$
\theta_{n}=O\left(L_{n}\right)
$$

and horocyclically if

$$
\theta_{n}^{2} / L_{n} \rightarrow 0
$$

If $\lambda_{n} / \lambda \rightarrow 1$ radially or horocyclically, we say the same is true for $\lambda_{n} \rightarrow \lambda$.

Now let $f_{n} \rightarrow f$ algebraically, and consider a parabolic point $c \in J(f)$ with period $i$. Suppose:

(a) The parabolic point $c$ has $p$ petals, and its multiplier $\lambda=\left(f^{i}\right)^{\prime}(c)$ is a primitive $p$ th root of unity;

(b) There are fixed-points $c_{n}$ of $f_{n}^{i}$ converging to $c$; and

(c) Their multipliers $\lambda_{n}=\left(f_{n}^{i}\right)^{\prime}\left(c_{n}\right)$ satisfy $\lambda_{n} \rightarrow \lambda$ radially (or horocyclically).

If these conditions hold for all parabolic points $c \in J(f)$, we say $f_{n} \rightarrow f$ radially (or horocyclically). (The formal definition $(\S 7)$ is somewhat more general.)

We say $f_{n} \rightarrow f$ preserving critical relations if for every critical point $b \in J(f)$ satisfying $f^{i}(b)=f^{j}(b)$, there are critical points $b_{n} \rightarrow b$ for $f_{n}$, with the same multiplicity as $b$, also satisfying the relation $f_{n}^{i}\left(b_{n}\right)=f_{n}^{j}\left(b_{n}\right)$.

In $\S 9$ and $\S 11$ we establish:

Theorem 1.3. Let $f$ be geometrically finite and let $f_{n} \rightarrow f$ horocyclically, preserving critical relations. Then $J\left(f_{n}\right) \rightarrow J(f)$ in the Hausdorff topology, and $f_{n}$ is geometrically finite for all $n \gg 0$.

Theorem 1.4. If, in addition,

(a) $f_{n} \rightarrow f$ radially, or

(b) H. $\operatorname{dim} J(f)>2 p(f) /(p(f)+1)$,

then $\mathrm{H} . \operatorname{dim} J\left(f_{n}\right) \rightarrow \mathrm{H} \cdot \operatorname{dim} J(f)$ and the canonical densities satisfy $\mu_{n} \rightarrow \mu$ in the weak topology on measures.

Here $p(f)$ is the maximum number of petals at a parabolic point of $f$ or one of its preimages $(\S 3)$. On the other hand we find $(\S 14)$ : 
Theorem 1.5. For any $\epsilon$ with $0<\epsilon<1 / 2$, there exist geometrically finite rational maps such that $f_{n} \rightarrow f$ horocyclically, preserving critical relations, but

$$
\text { H. } \operatorname{dim} J\left(f_{n}\right) \rightarrow 1>\text { H. } \operatorname{dim} J(f)=1 / 2+\epsilon .
$$

In these examples $p(f)=1$.

Quadratic polynomials. $\S 13$ presents the following applications of the continuity Theorem 1.4 to quadratic polynomials.

Corollary 1.6. If $\lambda$ is a root of unity, and $\lambda_{n} \rightarrow \lambda$ radially, then

$$
\text { H. } \operatorname{dim} J\left(\lambda_{n} z+z^{2}\right) \rightarrow \text { H. } \operatorname{dim} J\left(\lambda z+z^{2}\right) .
$$

Corollary 1.7. The function $\mathrm{H} . \operatorname{dim} J\left(z^{2}+c\right)$ is continuous for $c$ in the interval $\left(c_{\text {Feig }}, 1 / 4\right]$, where $c_{\text {Feig }}=-1.401155 \ldots$ is the Feigenbaum point .

Using geometric limits, the same methods show:

Theorem 1.8. Let $\lambda$ be a primitive pth root of unity. Then there exist $\lambda_{n} \rightarrow \lambda$ horocyclically such that

$$
\lim \inf \text { H. } \operatorname{dim} J\left(\lambda_{n} z+z^{2}\right) \geq \frac{2 p}{p+1} .
$$

This yields a new proof of a result of Shishikura:

Corollary 1.9. There exist expanding quadratic polynomials $f$ with H. $\operatorname{dim} J(f)$ arbitrarily close to 2.

Parallels with Kleinian groups. In Part I of this series we discuss related results for Kleinian groups. For example, work of Bishop and Jones [4], [28, Thm. 2.1 ] shows the radial limit set of any Kleinian group satisfies

$$
\alpha(\Gamma)=\mathrm{H} \cdot \operatorname{dim} \Lambda_{\mathrm{rad}}(\Gamma) .
$$

Our definition of the radial Julia set and Theorem 1.1 are formulated to extend this result to the dynamics of rational maps. Similarly Theorem 1.2 is modeled after a result of Sullivan on geometrically finite Kleinian groups [42], [28, Thm. 3.1].

Kleinian groups $\Gamma_{n}$ converge to $\Gamma$ strongly if the convergence is both algebraic and geometric. Versions of Theorems 1.3 and 1.4 also hold for strongly convergent sequences of Kleinian groups [28]. Thus the hypothesis of Theorem 1.3 is a reasonable candidate for the definition of strong convergence in the setting of ra- 
tional maps. In [28] we show strong convergence alone is insufficient to guarantee convergence of the dimension of the limit set of a Kleinian group, and Theorem 1.5 gives a similar counterexample for rational maps.

Many of our results are proved by a new method that reduces the study of parabolic fixed-points, their bifurcations and their geometric limits, to the case of elementary Kleinian groups. The reduction involves quasiconformal conjugacies with small conformal distortion; it is developed in $\S 6$ and $\S 7$.

The dimension lower bound of $2 p /(p+1)$ in Theorem 1.8 is related, via this method, to the well-known lower bound H. $\operatorname{dim} \Lambda>1$ for the limit set of a Kleinian group with a rank 2 cusp [28, Cor. 2.2]. In fact a suitable geometric limit of $f_{n}(z)=\lambda_{n} z+z^{2}$ behaves like a $p$-fold covering of a rank 2 cusp (§12).

To prove continuity of dimension when $f_{n} \rightarrow f$, we study the accumulation points $\nu$ of the canonical densities $\mu_{n}$ for $f_{n}$. By controlling the concentration of these densities, we show $\nu$ has no atoms, so by Theorem 1.4 it coincides with the canonical density for $f(\S 11)$. It follows that H. $\operatorname{dim} J\left(f_{n}\right) \rightarrow$ H. $\operatorname{dim} J(f)$.

Notes and references.The first equality in Theorem 1.1 is due to Denker, Urbański and Przytycki [11], [32], [36]. The second was observed independently in [46]. Basic references for the dynamics of rational maps include [3], [7], [30] and [40]. For the dictionary between rational maps and Kleinian groups, see [41] and $[25]$.

Several sections below include an exposition and consolidation of results known to experts, with references and remarks collected in notes at the end of each section. We hope the present systematic treatment will provide a useful contribution to the foundations of the field.

Part III of this series presents explicit dimension calculations for families of conformal dynamical systems.

Notation. $A \asymp B$ means $A / C<B<C A$ for some implicit constant $C$; $n \gg 0$ means for all $n$ sufficiently large.

\section{The basic invariants}

Let $f: \widehat{\mathbb{C}} \rightarrow \widehat{\mathbb{C}}$ be a rational map on the Riemann sphere. In this section we assemble results comparing:

- $\alpha(f)$, the minimum dimension of a $f$-invariant density on the Julia set;

- hyp-dim $(f)$, the sup of the dimensions of expanding subsets of the Julia set; and

- H. $\operatorname{dim} J_{\mathrm{rad}}(f)$, the Hausdorff dimension of the radial Julia set.

We assume throughout that $f$ has degree 2 or more. We also equip $\widehat{\mathbb{C}}$ with the 
spherical metric $2|d z| /\left(1+|z|^{2}\right)$ and let $\left|f^{\prime}(z)\right|$ denote the spherical derivative.

Definitions. The Julia set $J(f)$ is the closure of the set of repelling periodic points for $f$. The Fatou set is its complement, $\Omega(f)=\widehat{\mathbb{C}}-J(f)$.

The critical points of $f$ (where $f^{\prime}(c)=0$ ) form the critical set $C(f)$. The postcritical set is given by

$$
P(f)=\overline{\bigcup_{n=1}^{\infty} f^{n}(C(f)) .}
$$

The Herman-Siegel set $H S(f)$ is the union of the periodic Herman rings and Siegel disks for $f$.

The radial Julia set. We define the radial Julia set $J_{\text {rad }}(f)$ as follows. First, say $x$ belongs to $J_{\text {rad }}(f, r)$ if for any $\epsilon>0$, there is a neighborhood $U$ of $x$ and $n>0$ such that $\operatorname{diam}(U)<\epsilon$ and

$$
f^{n}: U \rightarrow B\left(f^{n}(x), r\right)
$$

is a homeomorphism. Then set

$$
J_{\operatorname{rad}}(f)=\bigcup_{r>0} J_{\operatorname{rad}}(f, r)
$$

We have $x \in J_{\text {rad }}(f)$ iff arbitrarily small neighborhoods of $x$ can be blown up univalently by the dynamics to balls of definite size centered at $f^{n}(x)$.

Invariants. An $f$-invariant density of dimension $\alpha$ is a positive measure $\mu$ on $\widehat{\mathbb{C}}$ such that

$$
\mu(f(E))=\int_{E}\left|f^{\prime}\right|^{\alpha} d \mu
$$

for every Borel set $E$ such that $f \mid E$ is injective. Thus $\mu$ transforms like a form of type $|d z|^{\alpha}$.

The critical dimension of $f$ is defined by

$$
\alpha(f)=\inf \{\alpha \geq 0: \exists \text { an } f \text {-invariant density on } J(f) \text { of dimension } \alpha\} .
$$

(One can also allow densities on $\widehat{\mathbb{C}}$; see Corollary 4.5). As for Kleinian groups, the infimum is achieved, and we have $\alpha(f)>0$ because there is no finite forwardinvariant measure for $f$.

Following [37], we say a compact set $X \subset \widehat{\mathbb{C}}$ is hyperbolic if $f(X) \subset X$ and 
$f$ is expanding on $X$. The latter condition means there exists an $n$ such that $\left|\left(f^{n}\right)^{\prime}(x)\right|>1$ for all $x \in X$. Equivalently, $\left\|f^{\prime}\right\|>1$ with respect to a smooth conformal metric $\rho$ defined near $X$, e.g. the metric

$$
\rho=\sigma+f^{*} \sigma+\cdots\left(f^{n-1}\right)^{*} \sigma
$$

where $\sigma$ is the spherical metric. Any hyperbolic set is contained in $J(f)$. The hyperbolic dimension of $f$ is defined by

$$
\operatorname{hyp}-\operatorname{dim}(f)=\sup \{\text { H. } \operatorname{dim} X: X \text { is a hyperbolic set for } f\} \text {. }
$$

We may now state:

Theorem 2.1. Any rational map $f$ of degree greater than one satisfies

$$
\alpha(f)=\operatorname{hyp}-\operatorname{dim}(f)=\text { H. } \operatorname{dim} J_{\text {rad }}(f) .
$$

The proof relies on work of Denker, Urbański and Przytycki, and some preliminaries on the radial Julia set.

Let $J_{\text {hyp }}(f)$ denote the union of the hyperbolic sets for $f$. By the expanding property it is easy to see:

Proposition 2.2. For any rational map $f, J_{\text {hyp }}(f) \subset J_{\text {rad }}(f)$.

Proposition 2.3. For any $r>0$ and $x \in J_{\text {rad }}(f, r)$, there are arbitrarily small balls $B(x, s)$ such that for any $f$-invariant density $\mu$ of dimension $\beta$,

$$
\mu(B(x, s)) \asymp s^{\beta} .
$$

The implicit constants are independent of $x$ and $s$.

Proof. By the definition of the radial Julia set and the Koebe distortion theorem, there are arbitrarily small $s$ such that $B(x, s)$ can be mapped by a suitable iterate $f^{n}$, univalently and with bounded distortion, to an open set $U \supset B\left(f^{n}(x), r / 10\right)$. We have $\mu(U) \asymp 1$ and $\left|\left(f^{n}\right)^{\prime}\right| \asymp 1 /|s|$ on $B(x, s)$, so (2.3) follows from the transition formula (2.2) for $\mu$.

Corollary 2.4. For any rational map $f$, H. $\operatorname{dim} J_{\text {rad }}(f) \leq \alpha(f)$.

Proof. Let $\mu$ be an $f$-invariant density of dimension $\alpha(f)$. Fix $r>0$; we will first show H. $\operatorname{dim} J_{\text {rad }}(f, r) \leq \alpha(f)$.

Fix $\epsilon>0$ and let $B\left(x_{1}, s_{1}\right)$ be a ball of maximum radius $s_{1} \leq \epsilon$ centered in 
$J_{\text {rad }}(f, r)$ and satisfying (2.3). Inductively define $B\left(x_{i}, s_{i}\right)$ to be a ball of maximum radius $s_{i} \leq \epsilon$, centered in $J_{\mathrm{rad}}(f, r)$, satisfying $(2.3)$ and disjoint from all the balls chosen so far. Then any ball $B(x, s)$ not chosen must meet one that was chosen, so we have

$$
J_{\text {rad }}(f, r) \subset \bigcup B\left(x_{i}, 3 s_{i}\right)
$$

(compare [39, I.3.1]). On the other hand, the chosen balls are disjoint, so

$$
\sum\left(\operatorname{diam} B\left(x_{i}, 3 s_{i}\right)\right)^{\alpha(f)} \asymp \sum \mu\left(B\left(x_{i}, s_{i}\right)\right) \leq \mu(J(f)) .
$$

This shows H. $\operatorname{dim}\left(J_{\text {rad }}(f, r)\right) \leq \alpha(f)$.

Since $J_{\operatorname{rad}}(f)=\bigcup J_{\text {rad }}(f, 1 / n)$ the same bound holds for the dimension of the radial Julia set.

Proof of Theorem 2.1. According to [36, Thm. 9.3.11] we have:

$$
\alpha(f) \leq \operatorname{hyp}-\operatorname{dim}(f) .
$$

On the other hand, the preceding results show

$$
\operatorname{hyp}-\operatorname{dim}(f) \leq \mathrm{H} \cdot \operatorname{dim} J_{\mathrm{hyp}}(f) \leq \mathrm{H} \cdot \operatorname{dim} J_{\mathrm{rad}}(f) \leq \alpha(f),
$$

so all these quantities agree.

\section{Notes.}

1. For results related to Theorem 2.1, see also [11], [32], [35], [46], [36].

2. The radial Julia set was defined independently by Urbański. Theorem 2.1 is stated in [46, p.21]; see also [10]. Various other possible definitions for the radial Julia set are investigated in [35].

3. Our definition of $J_{\text {rad }}(f)$ is intended as a translation, to the dynamical setting, of the definition of the radial limit set of a Kleinian group $\Gamma$. To see the analogy, recall that $x$ belongs to the radial limit set iff a geodesic ray $\rho \subset \mathbb{H}^{d+1}$ landing at $x$ projects to a recurrent geodesic on $M=\mathbb{H}^{d+1} / \Gamma$. This means there is a fixed compact set $K \subset \mathbb{H}^{d+1}$, a sequence $y_{n} \in \rho$ converging to $x$, and a sequence $\gamma_{n} \in \Gamma$ such that $\gamma_{n}\left(y_{n}\right) \in K$. Let $U_{n} \subset S_{\infty}^{d}$ be the sequence of round balls shrinking to $x$ cut off by the hyperplanes through $y_{n}$ normal to $\rho$. Since $\gamma_{n}$ moves $y_{n}$ into $K$, it blows up $U_{n}$ with bounded distortion to a ball of definite size, just as in the definition of $J_{\text {rad }}(f)$.

4. In general $J_{\text {rad }}(f)$ is strictly larger than $J_{\text {hyp }}(f)$. A nice example is furnished by the parabolic map $f(z)=z^{2}+1 / 4$.

In this case $J_{\text {hyp }}(f)$ is meager in $J(f)$. Indeed, liminf $\left|\left(f^{n}\right)^{\prime}(x)\right|^{1 / n}=1$ along a dense $G_{\delta}$ containing the inverse orbit of the parabolic fixed-point, while 
$\liminf \left|\left(f^{n}\right)^{\prime}(x)\right|^{1 / n}>1$ for any $x \in J_{\text {hyp }}(f)$. On the other hand, the radial limit set $J_{\text {rad }}(f)$ is almost all of $J(f)$; it only excludes the countable inverse orbit of the parabolic fixed-point (see Theorem 6.5 below).

5. Spectral theory. A density $\mu$ of dimension $\alpha$ on $\widehat{\mathbb{C}}$ determines, via visual extension, a positive function $\phi$ on $\mathbb{H}^{3}$ satisfying $\Delta \phi=\alpha(2-\alpha) \phi$. If $\mu$ is $f$-invariant, then $\phi$ descends to a positive eigenfunction on the 3-dimensional hyperbolic lamination $\mathcal{L}_{f}$ associated to $f$ by Lyubich and Minsky. Thus invariant densities should reflect the spectral geometry of $\mathcal{L}_{f}$ in the same way that invariant densities for a Kleinian group $\Gamma$ reflect the spectral geometry of the 3 -manifold $\mathbb{H}^{3} / \Gamma$ (compare $[23, \S 9.8]$ ).

\section{Petals and dimension}

In this section we briefly describe the effect of parabolic points on the critical dimension of a rational map $f$. We will establish:

Theorem 3.1. The petal number of $f$ bounds the critical dimension from below by

$$
\alpha(f)>\frac{p(f)}{p(f)+1} .
$$

Petal number. Let $c$ be a periodic point for $f$. Then $c$ is a parabolic point with $p>0$ petals if, for some $i>0, c$ is a fixed-point of $f^{i}$ of multiplicity $p+1$. This means there is a local coordinate with $z(c)=0$ such that

$$
f^{i}(z)=z+z^{p+1}+O\left(z^{p+2}\right) .
$$

The terminology comes from the 'Leau-Fatou flower theorem', which asserts that the immediate attracting basin of $c$ contains $p$ domains touching symmetrically at $c[30, \S 7],[7, \mathrm{II} .5],[40$, Ch. 3.5].

Now let $b$ be a critical point of $f$ whose forward orbit lands on a parabolic point $c$ with $p$ petals; say $f^{i}(b)=c$. Then $b$ is a preparabolic critical point with $d p$ petals, where $d$ is the local degree of $f^{i}$ at $b$. In this case we can replace $f$ by a finite iterate to arrange that $f(b)=c, f(c)=c$ and $f^{\prime}(c)=1$. Then in an appropriate coordinate with $z(b)=0$, we obtain a local parabolic fixed point for $g$ where

$$
g(z)=f^{-1} \circ f \circ f(z)=z+z^{d p+1}+O\left(z^{d p+2}\right) .
$$

The $d p$ petals of $g$ are just the preimages under $f$ of the $p$ petals at $c$. The dynamics of (3.2) near $b$ is semiconjugate, by the $d$-to- 1 map $f$, to the dynamics of 
(3.1) near $c$. There are $(d-1)$ choices for $g$, differing by the choice of the inverse branch $f^{-1}$.

The petal number $p(f)$ is the maximum of the number of petals at all parabolic points and all preparabolic critical points of $f$. We set $p(f)=0$ if no such points exist. Note that $p\left(f^{i}\right)=p(f)$ for any $i>0$.

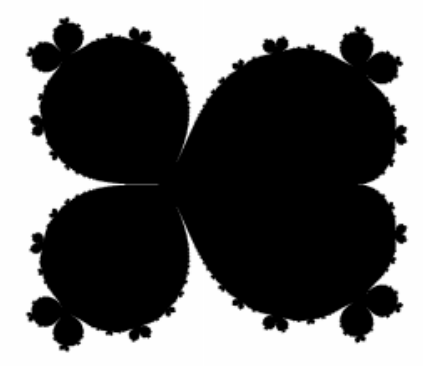

Figure 1. The filled Julia set of $f(z)=z(1+z)^{3}$ has three petals at $z=-1$.

Example. For $f(z)=z(1+z)^{d}$, we have $p(f)=d$. Although the parabolic point at $z=0$ has only one petal, the map $f$ also has a preparabolic critical point $b=-1$ of local degree $d$. Thus $f$ has $d$ petals at $b$ (see Figure 1 for the case $d=3$ ).

To begin the proof of Theorem 3.1, we show:

Proposition 3.2. If $f(z)$ has a parabolic point with $p$ petals, then $\alpha(f)>p /(p+1)$.

Proof. Replacing $f$ with a suitable iterate and making a change of coordinates, we can assume the parabolic point is at $z=\infty$ and

$$
f(z)=z+z^{1-p}+O\left(z^{-p}\right)
$$

Letting $w=z^{p}$ we obtain the (multi-valued) map in the $w$-plane

$$
f(w)=w+p+O\left(w^{-1 / p}\right)
$$

where the spherical metric $2|d z| /\left(1+|z|^{2}\right)$ becomes

$$
\sigma=\frac{2|d w|}{p\left(|w|^{1+1 / p}+|w|^{1-1 / p}\right)}
$$

Choose a point $w_{0} \in J(f)$ near $w=\infty$; then under the local dynamics, $w_{n}=$ 
$f^{-n}\left(w_{0}\right) \rightarrow \infty$ and in fact $\left|w_{n}\right| \asymp n$ by (3.3). On the other hand, $f^{\prime}(w)=$ $1+O\left(w^{-1-1 / p}\right)$; since $\prod\left(1+n^{-1-1 / p}\right)$ converges, by the chain rule we have $\left|\left(f^{-n}\right)^{\prime}\left(w_{0}\right)\right| \asymp 1$.

Now let $\mu$ be an $f$-invariant density of dimension $\alpha$. Then by considering the images $B_{n}=f^{-n}\left(B_{0}\right)$ of a small ball $B_{0}$ around $w_{0}$, we find

$$
\begin{aligned}
1 & \geq \sum \mu\left(B_{n}\right) \asymp \mu\left(B_{0}\right) \sum\left|\left(f^{-n}\right)^{\prime}\left(w_{0}\right)\right|_{\sigma}^{\alpha} \\
& \asymp \sum\left(\frac{1}{\left|w_{n}\right|^{1+1 / p}+\left|w_{n}\right|^{1-1 / p}}\right)^{\alpha} \asymp \sum n^{-\alpha(1+1 / p)},
\end{aligned}
$$

and for this last sum to converge we must have $\alpha>p /(p+1)$.

Proof of Theorem 3.1. It remains only to treat the case of a preparabolic critical point $b$. Replacing $f$ with a finite iterate $f^{i}$ (which does not change $p(f)$ or $\alpha(f)$ ), we can assume $f$ has local degree $d$ at $b, f(b)=c$ is a parabolic fixed-point with $p$ petals and $f^{\prime}(c)=1$. Let $g$ be a branch of $f^{-1} \circ f^{2}$ defined near $b$ as in (3.2). Since $g$ is contained in the full dynamics generated by $f$, it leaves invariant any $f$-invariant density $\mu$, and thus $\alpha(f)>d p /(d p+1)$ by the same argument as the preceding proof.

Note. Variants of Proposition 3.2 appear in [45, Thm. 7.14] and [1, Thm. 8.5].

\section{Poincaré series}

For $x \in \widehat{\mathbb{C}}$ we define the absolute Poincaré series by

$$
P_{s}(f, x)=\sum_{n=0}^{\infty} \sum_{f^{n}(y)=x}\left|\left(f^{n}\right)^{\prime}(y)\right|^{-s},
$$

the critical exponent at $x$ by

$$
\delta(f, x)=\inf \left\{s>0: P_{s}(f, x)<\infty\right\},
$$

and the critical exponent of $f$ by

$$
\delta(f)=\inf _{\widehat{\mathbb{C}}} \delta(f, x) .
$$

In this section we will establish:

Theorem 4.1. Suppose the critical exponent $\delta(f, x)$ is finite for some $x \in \widehat{\mathbb{C}}$. 
Then the Julia set carries an $f$-invariant density $\mu$ of dimension $\delta(f, x)$ with no atoms on the parabolic or repelling points of $f$, or any of their preimages.

Corollary 4.2. For any rational map, $\alpha(f) \leq \delta(f)$.

We begin with some preliminary remarks about the behavior of these Poincaré series. Recall from $\S 2$ that $P(f)$ denotes the postcritical set, $\Omega(f)$ the Fatou set, and $H S(f)$ the union of the Siegel disks and Herman rings for $f$.

Proposition 4.3. Let $x$ belong to the Fatou set of a rational map $f$. Then

- $\delta(f, x)=\infty \Longleftrightarrow x \in P(f) \cup H S(f)$.

Assuming $\delta(f, x)<\infty$ we also have:

- $\delta(f, x) \leq 2$,

- $P_{2}(f, x)<\infty$,

- $P_{\alpha}(f, x)<\infty$ if $x$ meets the support of an invariant density of dimension $\alpha$, and

- $f^{-n}(x) \rightarrow J(f)$ in the Hausdorff topology.

Proof. Assume $x$ is in the Fatou set. Suppose $x \in H S(f)$; then the terms in the Poincaré series do not tend to zero, so $\delta(f, x)=\infty$. If $x \in P(f)-H S(f)$, then $x$ is an attracting periodic point or some preimage of $x$ is a critical point; in either case $\delta(f, x)=\infty$.

Now suppose $x \notin P(f) \cup H S(f)$. Then $x$ is the center of a ball $B$ disjoint from both $P(f)$ and $\bigcup_{1}^{\infty} f^{n}(B)$. It follows that $f^{-n}$ is univalent on $B$, all the preimages of $B$ are disjoint and their total spherical area is finite, so $P_{2}(f, x)$ is also finite by the Koebe distortion theorem. In particular $\delta(f, x) \leq 2$. By the same token, $P_{\alpha}(f, x)$ is comparable to $\mu\left(\bigcup f^{-n}(B)\right)<\infty$. The convergence of the preimages of $x$ to $J(f)$ follows from the classification of stable regions.

Corollary 4.4. The Julia set of any rational map supports an invariant density of dimension $0<\alpha \leq 2$ with no atoms at the parabolic or repelling points of $f$, or any of their preimages.

Proof. If $J(f)=\widehat{\mathbb{C}}$ take $\mu$ to be Lebesgue area measure. Otherwise, the preceding Proposition shows there is an $x \notin J(f)$ with $\delta(f, x) \leq 2$, and Theorem 4.1 yields the desired density.

Corollary 4.5. One can also define $\alpha(f)$ as the infimum of the dimensions of all $f$-invariant densities on the sphere.

Proof. Let $\mu$ be an invariant density of dimension $\alpha_{0}$ with positive mass on the Fatou set. By invariance, the support of $\mu$ contains some $x \in \Omega(f)-(H S(f) \cup$ $P(f)$ ). Then $\delta(f, x) \leq \alpha_{0}$ by the preceding Proposition, and $J(f)$ supports an 
invariant density of dimension $\delta(f, x)$ by Theorem 4.1. Thus $\alpha(f) \leq \delta(f, x) \leq \alpha_{0}$.

Proof of Theorem 4.1. We begin by recalling the Patterson-Sullivan construction of an invariant density $\mu$ of dimension $\delta(f, x)$.

For $s>\delta(f, x)$ consider the probability measure

$$
\mu_{s}=\frac{1}{P_{s}(f, x)} \sum_{f^{n}(y)=x}\left|\left(f^{n}\right)^{\prime}(y)\right|^{-s} \delta_{y}
$$

where $\delta_{y}$ is the $\delta$-mass at $y$. Let $E$ be a Borel set with $f \mid E$ injective. Then by the chain rule

$$
\int_{E}\left|f^{\prime}\right|^{s}=\mu_{s}(f(E))+ \begin{cases}\left|f^{\prime}(x)\right|^{s} / P_{s}(f, x) & \text { if } x \in E \\ 0 & \text { otherwise. }\end{cases}
$$

If the Poincaré series diverges at the critical exponent, we let $\mu$ be an weak limit of $\mu_{s}$ as $s \searrow \delta(f, x)$.

If the Poincaré series converges at $s=\delta(f, x)$, we modify it to force $P_{s}(f, x) \rightarrow$ $\infty$. More precisely, as $s \rightarrow \delta(f, x)$ we change a large but finite number of terms from $\left|\left(f^{n}\right)^{\prime}(y)\right|^{s}$ to $\left|\left(f^{n}\right)^{\prime}(y)\right|^{t}$, where $t=2 \delta(f, x)-s$. Then (4.3) becomes, for $x \notin E$,

$$
\int_{E} \min \left(\left|f^{\prime}\right|^{s},\left|f^{\prime}\right|^{t}\right) d \mu_{s} \leq \mu_{s}(f(E)) \leq \int_{E} \max \left(\left|f^{\prime}\right|^{s},\left|f^{\prime}\right|^{t}\right) d \mu_{s}
$$

and $t \nearrow \delta(f, x)$ as $s \searrow \delta(f, x)$. Again we let $\mu$ be any weak limit of $\mu_{s}$.

The $f$-invariance of $\mu$ as a density of dimension $\delta(f, x)$ follows from (4.3) or (4.4), and $\mu$ is supported on $J(f)$ because $f^{-n}(x) \rightarrow J(f)$.

Atoms. Let $p \in J(f)$ be a repelling or parabolic periodic point, or one of its preimages. To complete the proof, we will show $\mu(p)=0$.

To begin, fix $\epsilon>0$. We will construct a neighborhood $U$ of $p$ such that

$$
\lim \sup \mu_{s}(U-p)<\epsilon
$$

The argument breaks into three cases, depending on whether $p$ is (I) repelling, (II) parabolic or (III) preperiodic.

Let $\delta=\delta(f, x)$ and $t=2 \delta-s$; note that $\delta \geq \alpha(f)>0$.

I. Repelling. Suppose $p$ is a repelling fixed-point. Then there is a sequence of fundamental annuli $A_{n}$ for the linearized dynamics, nesting down to $p$ and disjoint from $x$, such that $f^{n}: A_{n} \rightarrow A_{0}$ satisfies $\left|\left(f^{n}\right)^{\prime}\right| \asymp \lambda^{n}$ for some $\lambda>1$. By (4.4) we have

$$
\mu_{s}\left(A_{n}\right)=O\left(\lambda^{-n t} \mu_{s}\left(A_{0}\right)\right)=O\left(\lambda^{-n t}\right)
$$


since $\mu_{s}\left(A_{0}\right) \leq 1$. Letting $U=\{p\} \cup \bigcup_{N}^{\infty} A_{n}$ we have

$$
\mu_{s}(U-p)=O\left(\sum_{N}^{\infty} \lambda^{-n t}\right)<\epsilon
$$

for $N$ sufficiently large and all $s$ close enough to $\delta$. The case of a repelling periodic point is similar.

II. Parabolic. Now suppose $p$ is a parabolic fixed-point of $f$ with one petal. Then we can choose coordinates so that $p=0$ and

$$
f(z)=z+z^{2}+O\left(z^{3}\right)
$$

near $p$. Locally $f(z)$ behaves like the parabolic Möbius transformation $T(z)=$ $z /(1-z)$, and the Julia set is asymptotic to the positive real axis (cf. [7, II.5]).

Choose a fundamental domain $A_{0}$ for the dynamics $f$ near $J(f)$. The region $A_{0}$ can be taken to be approximately a square of size about $c^{2}$ centered at a point $c>0$, where $c$ is small. Then the Julia set near $z=0$ is covered by $\{p\} \cup A_{0} \cup A_{1} \cup \ldots$, where $f^{n}: A_{n} \rightarrow A_{0}$ and $d\left(0, A_{n}\right) \asymp c / n$. By choosing $A_{0}$ close to $p$ we can guarantee that all the $A_{n}$ are disjoint from $x$.

Since the parabolic point $p$ has one petal, by Theorem 3.1 we have $\delta>1 / 2$. The map $f^{n}$ on $A_{n}$ behaves like $T^{n}(z)=z /(1-n z)$, so $\left|\left(f^{n}\right)^{\prime}\right| \asymp(n c)^{2}$. Taking $U=\{p\} \cup \bigcup_{N}^{\infty} A_{n}$ we have

$$
\mu_{s}(U-p)=O\left(\sum_{N}^{\infty} \frac{1}{(n c)^{2 t}}\right)<\epsilon
$$

for $N$ sufficiently large and all $s$ close enough to $\delta$, since then $2 t>1$.

The case of a parabolic periodic point with more petals can be treated similarly, using e.g. the analysis in [7, II.5] or $\S 8$.

III. Preperiodic. Finally suppose $p$ is strictly preperiodic, with $q=f^{i}(p)=$ $f^{i+j}(p)$ a parabolic or repelling periodic point for some $i, j>0$. We must allow for the possibility that $p$ is a critical point of $f^{i}$; so suppose $f^{i}$ is locally $d$-to- 1 at $p$.

Consider the dynamical system

$$
g(z)=f^{-i} \circ f^{j} \circ f^{i}(z)
$$

defined by locally lifting the dynamics of $f^{j}$ from $q$ to $p$, so $g(p)=p$. There are $d$ choices for $g$, coming from cyclic permutations of the sheets of $f^{i}$.

Then on a punctured neighborhood $V$ of $p$, the measure $\mu_{s}$ transforms by (4.4) under $g$ as well as $f$, since $g$ is locally composed of univalent branches of $f$. Thus the preceding arguments yield a neighborhood $U$ of $p$ with $\mu_{s}(U-p)<\epsilon$. 
Conclusion. We have now constructed a neighborhood $U$ of $p$ with

$$
\lim \sup \mu_{s}(U-p)<\epsilon
$$

But we also have $\lim \sup \mu_{s}(p)=0$, since the Poincaré series for $\mu_{s}$ is constructed exactly so that the mass attached to any single term in the series tends to zero as $s \rightarrow \delta$. Thus $\mu(U) \leq \limsup \mu_{s}(U)<\epsilon$, and therefore $\mu(p)=0$.

Remark. It is easy to see that an invariant density $\mu$ must assign zero mass to a repelling fixed-point, because otherwise $\mu(p)=\left|f^{\prime}(p)\right|^{\delta} \mu(p)>\mu(p)$. But this argument does not show $\mu$ has zero mass on the inverse orbit of $p$, because the inverse orbit may contain a critical point. The treatment of repelling periodic points in the proof above was chosen to handle both cases the same way.

Notes. The Poincaré series construction of invariant densities was introduced by Patterson in the setting of Fuchsian groups [31], and applied to Kleinian groups and rational maps by Sullivan [41].

\section{Dynamics on the radial Julia set}

The measurable and topological dynamics of $f$ are particularly well-behaved when the radial Julia set supports an invariant density. In this section we show:

Theorem 5.1. There is at most one normalized $f$-invariant density $\mu$ supported on $J_{\text {rad }}(f)$. Any such measure is ergodic and of dimension $\alpha(f)$.

Theorem 5.2. If the radial Julia supports an invariant density $\mu$, then:

1. The Poincaré series $P_{s}(f, z)$ diverges at $s=\alpha(f)$ for all $z \in \mathbb{C}$;

2. Any Borel set $A \subset \widehat{\mathbb{C}}$ with $f(A) \subset A$ has zero or full $\mu$-measure; and

3. The forward orbit of $\mu$-almost every $z$ is dense in $J(f)$.

Proof of Theorem 5.1. Let $\nu$ be an $f$-invariant density of dimension $\beta$ supported on the radial Julia set, and let $\mu$ be an invariant density of dimension $\alpha(f)$.

Fix $r>0$. By Proposition 2.3, for any $x \in J_{\text {rad }}(f, r)$ there are arbitrarily small balls satisfying

$$
\frac{\nu(B(x, s))}{\mu(B(x, s))} \asymp \frac{s^{\beta}}{s^{\alpha(f)}}
$$

For $\beta>\alpha(f)$ this ratio tends to zero as $s \rightarrow 0$, and it follows that $\nu(J(f, r))=0$, contrary to our assumption that $\nu$ is supported on the radial Julia set. Thus $\beta=\alpha(f)$. 
The same argument shows any two invariant densities $\nu_{1}, \nu_{2}$ supported on $J_{\text {rad }}(f)$ are mutually absolutely continuous. If $E$ is an $f$-invariant set of positive $\nu$-measure, then $\nu \mid E$ is also an invariant density supported on $J_{\text {rad }}(f)$. Since $\nu \ll \nu \mid E$, the set $E$ has full $\nu$-measure thus $f$ is ergodic with respect to $\nu$.

Similarly, for any invariant $\nu_{1}, \nu_{2}$ supported on $J_{\text {rad }}(f)$, the Radon-Nikodym derivative $\phi=d \nu_{1} / d \nu_{2}$ is an $f$-invariant Borel function, hence constant by ergodicity. Thus there is at most one normalized invariant density supported on the radial Julia set.

Proof of Theorem 5.2.

1. Let us say $B^{\prime}$ is a descendant of a ball $B$ if for some $n>0, f^{n}: B^{\prime} \rightarrow B$ is a univalent map with bounded distortion. Choose $r>0$ such that $\mu\left(J_{\operatorname{rad}}(f, r)\right)>0$. By compactness of the Julia set, there are balls $\left\langle B_{1}, \ldots, B_{n}\right\rangle$ such that every $x \in J_{\text {rad }}(f, r)$ is contained in infinitely many descendants of $\left\langle B_{1}, \ldots, B_{n}\right\rangle$.

Let $A_{i} \subset J_{\text {rad }}(f, r)$ be the set of $x$ contained in infinitely many descendants of $B_{i}$. Then $\mu\left(A_{i}\right)>0$ for some $i$, and therefore

$$
\sum \mu\left(B^{\prime}\right)=\infty
$$

where the sum is over all descendants $B^{\prime}$ of $B_{i}$.

Now fix $x \in B_{i}$. Then any descendant $B^{\prime}$ of $B_{i}$ contains a point $y$ with $f^{n}(y)=x$, and

$$
\mu\left(B^{\prime}\right) \asymp\left|\left(f^{n}\right)^{\prime}(y)\right|^{-\alpha}
$$

where $\alpha=\alpha(f)$. Every such $y$ contributes to the Poincaré series $P_{\alpha}(f, x)$, and since $\sum \mu\left(B^{\prime}\right)=\infty$ we have $P_{\alpha}(f, x)=\infty$ for all $x \in B_{i}$.

Finally we show $P_{\alpha}(f, x)=\infty$ for all $x \in \widehat{\mathbb{C}}$. Clearly the Poincaré series diverges if the inverse orbit of $x$ meets a critical point of $f$. But if no critical point is encountered, the inverse orbit accumulates on $J(f)$, and so $x$ has a preimage $y$ in $B_{i}$. Then the preimages of $y$ contribute to the Poincaré series for $x$, and therefore $P_{\alpha}(f, x)=\infty$ in this case as well.

2. Let $A \subset J_{\text {rad }}(f)$ be a forward-invariant Borel set with $\mu(A)>0$. Let $x$ be a Lebesgue density point of $A$, so that

$$
\lim _{s \rightarrow 0} \frac{\mu(B(x, s) \cap A)}{\mu(B(x, s))}=1
$$

Since $x \in J_{\text {rad }}(f, r)$ for some $r>0$, there is a sequence $s_{n} \rightarrow 0$ and $k_{n} \rightarrow \infty$ such that

$$
f^{k_{n}}: B\left(x, s_{n}\right) \rightarrow D_{n}
$$


is a univalent, $f^{k_{n}}$ has bounded distortion and $D_{n} \supset B\left(f^{k_{n}}(x), r / 10\right)$. Now $f(A) \subset A$, so the density of $A$ in $D_{n}$ tends to 1 as $n \rightarrow \infty$. Pass to a subsequence such that $D_{n} \rightarrow D_{\infty}$ in the Hausdorff topology; then $\mu\left(D_{\infty} \cap A\right)=\mu\left(D_{\infty}\right)$. Since $D$ contains an open subset of $J(f)$, we have $f^{n}(D) \supset J(f)$ for some $n$, and thus $\mu\left(f^{n}(A)\right)=\mu(J(f))$. By forward invariance, $A$ has full measure in $J(f)$.

3. Choose a ball $B(x, r)$ centered on a point in the Julia set, and let $A$ be the set of $z \in J(f)$ whose forward orbits never enter $B(x, r)$. Then $A$ is forward invariant, and

$$
\mu(A) \leq \mu(J(f)-B(x, r))<\mu(J(f)) .
$$

By (2) we have $\mu(A)=0$, and thus the forward orbit of almost every $z \in J(f)$ enters $B(x, r)$. Since the Julia set has a countable base for its topology, $\mu$-almost every orbit is dense.

Note. For Theorem 5.1 see also [10].

\section{Geometrically finite rational maps}

A rational map $f$ is geometrically finite if $|P(f) \cap J(f)|<\infty$; equivalently, if every critical point in the Julia set is preperiodic. This condition rules out Siegel disks and Herman rings but permits parabolic cycles. (The postcritical set $P(f)$ is defined by (2.1).)

In this section we prove:

Theorem 6.1. Let $f$ be a geometrically finite rational map. Then

$$
\delta(f)=\text { H. } \operatorname{dim} J_{\text {rad }}(f)=\text { H. } \operatorname{dim} J(f)=\alpha(f) .
$$

Moreover $\widehat{\mathbb{C}}$ carries a unique normalized $f$-invariant density $\mu$ of dimension $\delta(f)$; the measure $\mu$ is nonatomic and supported on the radial Julia set; and the Poincaré series $P_{s}(f, x)$ diverges at $s=\delta(f)$ for any $x \in \widehat{\mathbb{C}}$.

We refer to the unique normalized density of dimension $\delta(f)$ as the canonical density for a geometrically finite rational map $f$.

Corollary 6.2. If $f$ is geometrically finite then $J(f)=\widehat{\mathbb{C}}$ or $\mathrm{H} \cdot \operatorname{dim}(J(f))<2$.

Proof. Otherwise Lebesgue measure on the sphere would be a second invariant density of dimension $\delta(f)=\mathrm{H} \cdot \operatorname{dim} J(f)=2$.

Corollary 6.3. If $f^{-1}(C)=C$ for some circle $C$, then $f$ is geometrically finite 
and either $J(f)=C$ or $\mathrm{H} . \operatorname{dim} J(f)<1$.

Proof. Clearly $f$ has no critical points in $C$ and $J(f) \subset C$, so $f$ is geometrically finite. If $J(f) \neq C$, then we can find a small interval $I \subset C-J(f)$ with disjoint preimages. Letting $\mu$ denote 1-dimensional Hausdorff measure, we have

$$
\int_{I} P_{1}(f, x) d \mu=\sum_{0}^{\infty} \mu\left(f^{-i}(I)\right) \leq \mu(C)<\infty,
$$

so $P_{1}(f, x)<\infty$ for almost every $x \in I$. Since the Poincaré series diverges at the critical exponent $\delta(f)=\mathrm{H} \cdot \operatorname{dim} J(f)$, we have H. $\operatorname{dim} J(f)<1$.

Remark. For $f$ in the preceding Corollary, either $f$ or $f^{2}$ is conjugate to a Blaschke product.

We begin the proof of Theorem 6.1 with:

Lemma 6.4. If $f$ is geometrically finite then $\delta(f) \leq 2$.

Proof. When $J(f) \neq \widehat{\mathbb{C}}$ this follows from Proposition 4.3 .

Now suppose $J(f)=\widehat{\mathbb{C}}$. Let $B \subset \widehat{\mathbb{C}}-P(f)$ be a spherical ball. Then there is a $\lambda<1$ such that

$$
\operatorname{diam}\left(B^{\prime}\right)=O\left(\lambda^{n}\right)
$$

for any component $B^{\prime}$ of $f^{-n}(B)$. Indeed, the sphere admits an orbifold metric $\rho$ with respect to which $\left\|f^{\prime}\right\|>C>1[44],[24, \S \mathrm{A}]$. Thus $B^{\prime}$ is exponentially small in the $\rho$-metric. But $\rho$ has singularities on $P(f)$ locally of the form $|d z| /|z|^{\alpha}$, $0<\alpha<1$, so the identity map is Hölder continuous from the $\rho$-metric to the spherical metric. Therefore the spherical diameter of $B^{\prime}$ is also exponentially small.

By the Koebe distortion theorem,

$$
\frac{1}{\left|\left(f^{n}\right)^{\prime}(y)\right|} \asymp \frac{\operatorname{diam}\left(B^{\prime}\right)}{\operatorname{diam}(B)}=O\left(\lambda^{-n}\right)
$$

for $y \in B^{\prime}$. Letting $\sigma$ denote spherical area measure, for any $\epsilon>0$ and $n \geq 0$ fixed, we have

$$
\int_{B} \sum_{y: f^{n}(y)=x}\left|\left(f^{n}\right)^{\prime}(y)\right|^{-2-\epsilon} d \sigma(x) \leq \operatorname{area}\left(f^{-n}(B)\right) \sup _{f^{-n}(B)}\left|\left(f^{n}\right)^{\prime}(y)\right|^{-\epsilon}=O\left(\lambda^{-n \epsilon}\right) .
$$


Summing over $n$, we find

$$
\int_{B} P_{2+\epsilon}(f, x) d \sigma(x)=O\left(\sum \lambda^{-n \epsilon}\right)<\infty .
$$

Thus $\delta(f, x) \leq 2$ for a.e. $x \in B$ and therefore $\delta(f) \leq 2$.

Theorem 6.5. Let $f$ be a geometrically finite rational map. Then $J(f)-J_{\text {rad }}(f)$ consists exactly of the inverse orbits of the parabolic points and critical points in the Julia set. In particular $J(f)-J_{\text {rad }}(f)$ is countable.

Proof. If $x$ belongs to $J_{\text {rad }}$, then limsup $\left|\left(f^{n}\right)^{\prime}(x)\right|=\infty$, so the forward orbit of $x$ contains no critical points or parabolic points.

Conversely, assume the forward orbit of $x \in J(f)$ contains no critical or parabolic points; we will show $x \in J_{\text {rad }}(f)$.

Suppose the forward orbit of $x$ meets $P(f)$. Every point in the finite set $P(f) \cap J(f)$ either lands on a parabolic or repelling cycle. Thus $x$ lands on a repelling cycle and therefore $x \in J_{\text {rad }}$.

Now suppose the forward orbit of $x$ is disjoint from $P(f)$. Whenever the forward orbit of $x$ comes near $P(f)$, it is pushed away from $P(f)$ by the dynamics of one of a finite number of parabolic or repelling cycles. Thus $s=\liminf d\left(f^{n}(x), P(f)\right)>$ 0 . Since all branches of $f^{-n}$ are univalent outside of $P(f)$, we obtain infinitely univalent maps $f^{-n}: B\left(f^{n}(x), s\right) \rightarrow V_{n}$ where $x \in V_{n}$. Letting $r=s / 2$ we obtain infinitely many maps $f^{n}: U_{n} \rightarrow B\left(f^{n}(x), r\right)$ such that $\operatorname{diam}\left(U_{n}\right) \asymp\left|\left(f^{n}\right)^{\prime}(x)\right|^{-1}$ by the Koebe distortion theorem.

Excluding the easy case of $f(z)=z^{n}$, we also know $\left\|\left(f^{n}\right)^{\prime}(x)\right\| \rightarrow \infty$ with respect to the Poincaré metric on $\widehat{\mathbb{C}}-P(f)[24$, Thm. 3.6]. Since the spherical and Poincaré metrics are comparable away from $P(f), \operatorname{diam}\left(U_{n}\right) \rightarrow 0$ and therefore $x \in J_{\mathrm{rad}}$.

Proof of Theorem 6.1. By Lemma 6.4, $\delta(f)$ is finite. Choose any $x \in \widehat{\mathbb{C}}$ with $\delta(f, x)<\infty$. By Theorem 4.1, there is an invariant density $\mu$ on $J(f)$ of dimension $\delta(f, x)$ with no atoms on the preperiodic points. Hence $\mu$ is supported on $J_{\text {rad }}(f)$.

We claim

$$
\delta(f)=\alpha(f)=\text { H. } \operatorname{dim} J_{\text {rad }}(f)=\text { H. } \operatorname{dim} J(f) .
$$

Indeed, any invariant density supported on $J_{\text {rad }}(f)$, such as $\mu$, has dimension $\alpha(f)$ by Theorem 5.1. Thus $\delta(f, x)=\alpha(f)$, and since this holds for all $x$ with finite critical exponent we have $\delta(f)=\alpha(f)$. The equality $\alpha(f)=\mathrm{H} \cdot \operatorname{dim} J_{\mathrm{rad}}(f)$ holds for all rational maps (Theorem 2.1), and H. $\operatorname{dim} J_{\text {rad }}(f)=$ H. $\operatorname{dim} J(f)$ since $J(f)-J_{\text {rad }}(f)$ is countable.

Since the radial Julia set supports an invariant density, the Poincaré series 
$P_{s}(f, x)$ diverges at $s=\delta(f)$ for all $x \in \widehat{\mathbb{C}}$ by Theorem 5.2.

Finally consider any normalized $f$-invariant density $\nu$ on $\widehat{\mathbb{C}}$ of dimension $\delta(f)$. We claim $\nu=\mu$.

To begin with, $\nu$ is nonatomic and supported on $J(f)$. Indeed, if $\nu$ had an atom, it would have an atom at a nonperiodic point $x \notin P(f)$, and for $\delta=\delta(f)$ we would have

$$
P_{\delta}(f, x)=\sum_{f^{n}(y)=x}\left|\left(f^{n}\right)^{\prime}(y)\right|^{-\delta}=\sum \nu(y) / \nu(x) \leq \nu(\widehat{\mathbb{C}}) / \nu(x)<\infty,
$$

contrary to the divergence of the Poincaré series at the critical exponent. Similarly, if the support of $\nu$ were to meet the Fatou set, we would have $P_{\delta}(f, x)<\infty$ for some $x \notin J(f)$ by Proposition 4.3, again contradicting divergence.

Since $J(f)-J_{\text {rad }}(f)$ is countable, $\nu$ is supported on the radial Julia set. But the radial Julia set carries at most one normalized invariant density (Theorem 5.1), so $\nu=\mu$.

Corollary 6.6. Any normalized invariant density supported on the Julia set of a geometrically finite rational map is either:

- the canonical density of dimension $\delta(f)$, or

- an atomic measure of dimension $\alpha>\delta(f)$ supported on the inverse orbits of parabolic points and critical points.

Proof. An invariant density of dimension $\alpha>\delta(f)$ must be supported on the countable set $J(f)-J_{\text {rad }}(f)$ by Theorem 5.1. By the transformation rule (2.2) it vanishes on the forward orbit of any critical point.

A rational map $f$ is expanding if $J(f)$ itself is a hyperbolic set. It is not hard to see $f$ is expanding $\Longleftrightarrow J(f) \cap P(f)=\emptyset \Longleftrightarrow J(f)=J_{\text {rad }}(f)$. Compare [24, Thm. 3.13]. Since the Julia set of an expanding map contains no critical points or parabolic cycles, we have:

Corollary 6.7. The Julia set of an expanding rational map $f$ supports a unique normalized $f$-invariant density.

\section{Notes.}

1. The existence and uniqueness of the invariant density $\mu$ for an expanding map was shown in [41].

2. The canonical density $\mu$ for a geometrically finite rational map can be related to Hausdorff and packing measures on $J(f)$ by the results of [45], which also gives Corollary 6.2. A generalization of Theorem 6.5 to mappings with nonrecurrent critical points is implicit in [45, Prop. 6.1]. Geometrically finite maps without critical points in $J(f)$ are studied in [12], and [1]. 
3. A topological classification of geometrically finite rational maps, akin to Thurston's classification of critically finite maps [15], has been given by Cui [9].

4. If $f$ is geometrically finite but not expanding, then $J(f)$ carries atomic $f$ invariant densities of any dimension $s>\delta(f)$. For example, if $x \in J(f)-P(f)$ is a critical point, then the density $\mu_{s}$ defined by (4.2) is $f$-invariant by (4.3). Similarly, if the forward orbit of $x \in J(f)-P(f)$ lands on a parabolic cycle, then we can augment $\mu_{s}$ by finitely many atoms along the forward orbit of $x$ to obtain an invariant density of dimension $s$.

5. It is natural to ask if

$$
\text { H. } \operatorname{dim} J_{\text {rad }}(f)=\text { H. } \operatorname{dim} J(f)
$$

for all rational maps $f$, and equality has been verified in several cases [34], [20]. For geometrically finite maps, equality follows from Theorem 6.1 above.

6. Among geometrically infinite quadratic polynomials, there are nearly parabolic examples where H. $\operatorname{dim} J(f)=\operatorname{hyp}-\operatorname{dim}(f)=2$ [38], [37]. On the other hand, H. $\operatorname{dim} J(f)<2$ for:

- maps with no recurrent critical points [45], [8];

- Collet-Eckmann maps [33], [19].

- the Fibonacci map [20]; and

- the quadratic maps with Siegel disks $f(z)=e^{2 \pi i \theta} z+z^{2}$, where $\theta$ is an irrational of bounded type [27].

One also has area $(J(f))=0$ if $f$ has no indifferent cycle and is not infinitely renormalizable [22].

\section{Creation of parabolics}

To study limits of rational maps, we need to understand the creation of parabolic points. Our prototype for this process is the sequence of maps

$$
f_{n}(z)=\lambda_{n} z+z^{p+1}
$$

converging to $f(z)=z+z^{p+1}$. The limit has a parabolic fixed-point with $p$ petals at the origin. This prototype arises generically for $f_{n}=g_{n}^{p}$ when the multiplier at a fixed point of $g_{n}$ tends to a $p$ th root of unity (see Proposition 7.3).

Since we will be interested in putting the local dynamics into this standard form, we will work with germs of analytic maps.

Maps with fixed points. Let $\mathcal{G}$ be the union, over all open regions $U \subset \widehat{\mathbb{C}}$, of all holomorphic maps $f: U \rightarrow \widehat{\mathbb{C}}$. Let $U(f)$ denote the domain of $f \in \mathcal{G}$. We say 
$f_{n} \rightarrow f$ in $\mathcal{G}$ if for any compact $K \subset U(f)$, we have $K \subset U\left(f_{n}\right)$ for all $n \gg 0$ and $f_{n}|K \rightarrow f| K$ uniformly. This definition makes $\mathcal{G}$ into a non-Hausdorff topological space.

Define the space $\mathcal{F} \subset \mathcal{G} \times \widehat{\mathbb{C}}$ of maps with fixed-points by

$$
\mathcal{F}=\{(f, c): c \in U(f) \text { and } f(c)=c\} .
$$

We give $\mathcal{F}$ the product topology.

Petals. For $(f, c) \in \mathcal{F}$ let $\operatorname{mult}(f, c)$ denote the multiplicity of the fixed-point $c$. Then $\operatorname{mult}(f, c)=r>1$ iff

$$
\begin{aligned}
f^{\prime}(c) & =1, \\
f^{(i)}(c) & =0 \text { for } 1<i<r, \text { and } \\
f^{(r)}(c) & \neq 0 .
\end{aligned}
$$

In this case we say $(f, c)$ is parabolic with $p=r-1$ petals, following the terminology of $\S 3$. Note that any iterate of $f$ has the same number of petals as $f$.

Dominant convergence. Suppose $\left(f_{n}, c_{n}\right) \rightarrow(f, c)$ in $\mathcal{F}, f^{\prime}(c)=1$, and $\operatorname{mult}(f, c)=r$. We say $\left(f_{n}, c_{n}\right) \rightarrow(f, c)$ dominantly if there exists an $M$ such that

$$
\left|f_{n}^{(i)}\left(c_{n}\right)\right| \leq M\left|f_{n}^{\prime}\left(c_{n}\right)-1\right| \text { for } 1<i<r
$$

The terminology is meant to suggest that the first derivative dominates the higherorder derivatives. The derivatives should be taken in a local chart around $c$. The dominance condition is automatic if $(f, c)$ has only one petal.

Roots of unity. More generally we say $(f, c) \in \mathcal{F}$ is parabolic if $f^{\prime}(c)=\lambda$ is a root of unity, say $\lambda^{q}=1$. Then we say $(f, c)$ has $p$ petals if $\left(f^{q}, c\right)$ does, and $\left(f_{n}, c_{n}\right) \rightarrow(f, c)$ dominantly if $f_{n} \rightarrow f$ in $\mathcal{G}$ and $\left(f_{n}^{q}, c_{n}\right) \rightarrow\left(f^{q}, c\right)$ dominantly.

Finally if $f^{\prime}(c)$ is not a root of unity, we adopt the convention that any sequence $\left(f_{n}, c_{n}\right) \rightarrow(f, c)$ converging in $\mathcal{F}$ does so dominantly.

Coordinate change. We say $\left(g_{n}, d_{n}\right) \rightarrow(g, d)$ is related to $\left(f_{n}, c_{n}\right) \rightarrow(f, c)$ by a coordinate change if there are bijective maps $\phi_{n} \rightarrow \phi$ in $\mathcal{G}$ such that the new sequence is obtain from the old one by conjugation: that is, such that

$$
\begin{aligned}
\left(g_{n}, d_{n}\right) & =\left(\phi_{n} \circ f_{n} \circ \phi_{n}^{-1}, \phi_{n}\left(c_{n}\right)\right), \\
(g, d) & =\left(\phi \circ f \circ \phi^{-1}, \phi(c)\right) .
\end{aligned}
$$


Proposition 7.1. Dominant convergence is preserved by a coordinate change.

Proof. The proposition is clear for a coordinate change by translation, such as $\phi_{n}(z)=z-c_{n}$. Thus we may assume $c_{n}=d_{n}=0$. We may also assume $f^{\prime}(0)=1$, since the case where $f^{\prime}(0)$ is a root of unity reduces to this case.

Let $\lambda_{n}=f_{n}^{\prime}(0) \rightarrow 1, r=\operatorname{mult}(f, 0)$. Write

$$
f_{n}(z)=z+z \epsilon_{n}(z)+O\left(z^{r}\right)
$$

where $\epsilon_{n}(z)$ is a polynomial of degree $r-2$ with coefficients bounded by $M\left|\lambda_{n}-1\right|$. Letting $\zeta=\phi_{n}(z)$, we have

$$
\begin{aligned}
g_{n}(\zeta) & =\phi_{n}\left(f_{n}(z)\right) \\
& =\phi_{n}(z)+\phi_{n}^{\prime}(z) \epsilon_{n}(z) z+\cdots+\frac{\phi_{n}^{(r-1)}(z)}{(r-1) !} \epsilon_{n}(z)^{r-1} z^{r-1}+O\left(z^{r}\right) \\
& =\zeta+a_{1} z+a_{2} z^{2}+\cdots=\lambda_{n} \zeta+b_{2} \zeta^{2}+b_{3} \zeta^{3}+\cdots
\end{aligned}
$$

Now for $1 \leq i<r$, an $\epsilon_{n}(z)$ occurs in each term contributing to $a_{i}$, so $\left|a_{i}\right|=$ $O\left(\left|\lambda_{n}-1\right|\right)$. Substituting $z=\phi_{n}^{-1}(\zeta)$, we find $\left|b_{i}\right| \leq M^{\prime}\left|\lambda_{n}-1\right|$ for $1<i<r$, where $M^{\prime}$ depends only on $M$ and bounds on the power series for $\phi_{n}$ and $\phi_{n}^{-1}$. Thus $\left(g_{n}, 0\right) \rightarrow(g, 0)$ dominantly.

Theorem 7.2. (Dominant normal form) Suppose $\left(f_{n}, c_{n}\right) \rightarrow(f, c)$ dominantly, and $\operatorname{mult}(f, c)=r>1$. Then after passing to a subsequence and making a coordinate change, we can assume $c_{n}=c=0$ and

$$
\begin{aligned}
f_{n}(z) & =\lambda_{n} z+z^{r}+O\left(z^{r+1}\right), \\
f(z) & =z+z^{r}+O\left(z^{r+1}\right) .
\end{aligned}
$$

Proof. First change coordinates so $c_{n}=c=0$. Consider the least $s$ in the range $1<s<r$ such that $f_{n}^{(s)}(0) \neq 0$ for all $n$ sufficiently large. Write

$$
f_{n}(z)=\lambda_{n} z+A_{n} z^{s}+O\left(z^{s+1}\right) .
$$

Let $\phi_{n}(z)=z-B_{n} z^{s}$ where

$$
B_{n}=\frac{A_{n}}{\lambda_{n}\left(\lambda_{n}^{s-1}-1\right)}
$$

Since $\left|A_{n}\right|=O\left(\left|\lambda_{n}-1\right|\right)$ by the definition of dominant convergence, and $\lambda_{n} \rightarrow 1$, we find $B_{n}=O(1)$. Thus $\phi_{n}$ is injective on a uniform neighborhood of $z=0$, and 
we can pass to a subsequence such that $\phi_{n} \rightarrow \phi$. Changing coordinates by $\phi_{n}$, we find $f_{n}$ becomes

$$
f_{n}(z)=\lambda_{n} z+\left(A_{n}+\lambda_{n} B_{n}-\lambda_{n}^{s} B_{n}\right) z^{s}+O\left(z^{s+1}\right)
$$

so by (7.1) the coefficient of $z^{s}$ now vanishes. After the coordinate change the convergence is still dominant, so we can continue the discussion replacing $s$ with $s+1$. After a finite number of coordinate changes we obtain $f_{n}(z)=\lambda_{n} z+A_{n} z^{r}+$ $O\left(z^{r+1}\right)$ and $f(z)=z+A z^{r}+O\left(z^{r+1}\right)$.

Since $\operatorname{mult}(f, z)=r$, we have $A \neq 0$, so a final linear change of coordinates renders $A_{n}=A=1$.

Proposition 7.3. Suppose $(f, c)$ is parabolic with petals and $f^{\prime}(c)=\lambda$ is a primitive pth root of unity. Then any sequence $\left(f_{n}, c_{n}\right) \rightarrow(f, c)$ in $\mathcal{F}$ converges dominantly.

Proof. We may assume $c_{n}=c=0$. Let $\lambda_{n}=f_{n}^{\prime}(0)$. We claim there is a coordinate change $\phi_{n} \rightarrow \phi$, fixing the origin, such that

$$
f_{n}(z)=\lambda_{n} z+O\left(z^{p+1}\right)
$$

for all $n \gg 0$.

This coordinate change is constructed by the same method as in the previous proof. Let $s$ increase from $s=2$ to $s=p$. For each fixed value of $s$, we apply a coordinate change of the form $\phi_{n}(z)=z \mapsto z-B_{n} z^{s}$ to kill the coefficient of $z^{s}$ in $f_{n}$. Since $f_{n} \rightarrow f$, the numerator $A_{n}$ in (7.1) converges; and the denominator has a nonzero limit because $\lambda^{s-1} \neq 1$. Thus $\phi_{n}$ tends to a limiting coordinate change $\phi$ as $n \rightarrow \infty$, and the composition of these for $2 \leq s \leq p$ puts $f_{n}$ into the form $(7.2)$.

From (7.2) we have

$$
f_{n}^{p}(z)=\lambda_{n}^{p} z+O\left(z^{p+1}\right)
$$

so $\left(f_{n}^{p}, c_{n}\right) \rightarrow\left(f^{p}, c\right)$ dominantly.

The next result is useful for handling preparabolic critical points.

Proposition 7.4. Suppose $\left(f_{n}, 0\right) \rightarrow(f, 0)$ dominantly, and $\left(g_{n}, 0\right) \rightarrow(g, 0)$ satisfies

$$
g_{n}(z)^{d}=f_{n}\left(z^{d}\right)
$$

Then $\left(g_{n}, 0\right) \rightarrow(g, 0)$ dominantly. 
Proof. We treat the main case, where $g^{\prime}(0)=f^{\prime}(0)=1$ and $(f, 0)$ has $p$ petals; then $(g, 0)$ has $d p$ petals. Applying a coordinate change to $f_{n} \rightarrow f$, and applying its pullback under $z \mapsto z^{d}$ to $g_{n} \rightarrow g$, we can assume $f_{n}(z)=\lambda_{n} z\left(1+O\left(z^{p}\right)\right)$. Then by (7.3),

$$
g_{n}(z)=f_{n}\left(z^{d}\right)^{1 / d}=\lambda_{n}^{1 / d} z\left(1+O\left(z^{d p}\right)\right),
$$

so $\left(g_{n}, 0\right) \rightarrow(g, 0)$ dominantly.

\section{Linearizing parabolic dynamics}

In this section we show that if $f(z)$ has a parabolic fixed-point with one petal at $z=\infty$, then $f$ is almost conformally conjugate to the translation $T(z)=z+1$. Similarly, a parabolic bifurcation $f_{n} \rightarrow f$ can be reduced to the model $T_{n} \rightarrow T$ where $T_{n}(z)=\lambda_{n} z+1$ and $\lambda_{n} \rightarrow 1$.

As these model mappings are Möbius transformations, we obtain a reduction of analytic dynamics to the theory of elementary Kleinian groups, modulo an almost conformal change of coordinates. This reduction simplifies the study of the Julia set and its dimension in the presence of parabolics.

We present these reductions as the following three successively more general theorems. In all three theorems the conjugacies fix $z=\infty$.

Theorem 8.1. Let

$$
f(z)=z+1+O(1 / z)
$$

be the germ of an analytic map with a parabolic fixed-point at $z=\infty$. Then for any $\epsilon>0, f$ is $(1+\epsilon)$-quasiconformally conjugate near $\infty$ to

$$
T(z)=z+1
$$

Theorem 8.2. Let $f_{n} \rightarrow f$ on a neighborhood of $z=\infty$ where

$$
\begin{aligned}
f_{n}(z) & =\lambda_{n} z+1+O(1 / z), \\
f(z) & =z+1+O(1 / z),
\end{aligned}
$$

and $\lambda_{n} \rightarrow 1$ horocyclically. Then for any $\epsilon>0$, there are $(1+\epsilon)$-quasiconformal maps $\phi_{n} \rightarrow \phi$ defined near $\infty$ and conjugating $f_{n} \rightarrow f$ to $T_{n} \rightarrow T$, where

$$
\begin{aligned}
T_{n}(z) & =\lambda_{n} z+1, \\
T(z) & =z+1 .
\end{aligned}
$$


Theorem 8.3. Let $f_{n} \rightarrow f$ on a neighborhood of $z=\infty$ where

$$
\begin{aligned}
f_{n}(z) & =\lambda_{n} z+z^{1-p}+O\left(1 / z^{p}\right), \\
f(z) & =z+z^{1-p}+O\left(1 / z^{p}\right),
\end{aligned}
$$

$p \geq 1$ and $\lambda_{n} \rightarrow 1$ horocyclically. Then for any $\epsilon>0$, there are $(1+\epsilon)$ quasiconformal maps $\phi_{n}, \phi$ defined near $\infty$ and conjugating $f_{n} \rightarrow f$ to $T_{n} \rightarrow T$, where

$$
\begin{aligned}
T_{n}(z) & =\lambda_{n}\left(z^{p}+1\right)^{1 / p}, \\
T(z) & =\left(z^{p}+1\right)^{1 / p} .
\end{aligned}
$$

After passing to a subsequence we can assume $\phi_{n} \rightarrow \phi$.

Terminology and remarks.

Horocyclic and radial convergence. Let $\lambda_{n} \rightarrow 1$ in $\mathbb{C}^{*}$, where $\lambda_{n}=\exp \left(L_{n}+\right.$ $\left.i \theta_{n}\right)$ with $\theta_{n} \rightarrow 0$. We say $\lambda_{n} \rightarrow 1$ radially if

$$
\theta_{n}=O\left(\left|L_{n}\right|\right)
$$

and horocyclically if

$$
\theta_{n}^{2} / L_{n} \rightarrow 0
$$

(In either case we also allow $\lambda_{n}=1$.)

In terms of hyperbolic geometry, radial convergence means $t_{n}=i\left|L_{n}\right|+\theta_{n}$ stays within a bounded distance of a geodesic landing at 0 in the upper half-plane, while horocyclic convergence means any horoball resting on $t=0$ in $\mathbb{H}$ contains all but finitely many terms in the sequence $\left\langle t_{n}\right\rangle$. Horocyclic convergence also means the complex torus $X_{n}=\mathbb{C}^{*} / \lambda_{n}^{\mathbb{Z}}$ converges to a cylinder as $n \rightarrow \infty$. More precisely, $\lambda_{n} \rightarrow 1$ horocyclically iff the generator of $\pi_{1}\left(\mathbb{C}^{*}\right) \subset \pi_{1}\left(X_{n}\right)$ is represented by an annulus $A_{n} \subset X_{n}$ with $\bmod A_{n} \rightarrow \infty$ as $n \rightarrow \infty$.

Holomorphic index. The holomorphic index of a fixed point $p$ of $f$ with multiplier $\lambda$ is given by

$$
\operatorname{ind}(f, p)=\operatorname{Res}_{p}\left(\frac{d z}{z-f(z)}\right)=\frac{1}{1-\lambda}
$$

The index satisfies $\sum_{f(p)=p} \operatorname{ind}(f, p)=1$ (see $\left.[30, \S 9]\right)$. Another characterization of horocyclic convergence, suggested by Shishikura, is that the real part of the holomorphic index tends to infinity; that is, $\lambda_{n} \rightarrow 1$ horocyclically iff 
$\left|\operatorname{Re}\left(1-\lambda_{n}\right)^{-1}\right| \rightarrow \infty$

Analytic obstructions. It is known that a germ $f(z)=z+1+O(1 / z)$ as in Theorem 8.1 has infinitely many moduli providing obstructions to a conformal conjugacy to $T(z)=z+1$ near $z=\infty$ [47].

In Theorem 8.2, a typical sequence $f_{n}$ should be thought of as a bifurcation in which the parabolic fixed-point $z=\infty$ for $f$ splits into a pair of repelling and attracting points for $f_{n}$. The domain of $\phi_{n}$ includes both points for $n$ large. Since the multipliers of $f_{n}$ and $T_{n}$ at their second fixed-points $(\neq \infty)$ generally differ, at best a quasiconformal conjugacy can be achieved.

Models for multiple petals. In Theorem 8.3, the $p$ th roots in the equations for $T_{n}$ and $T$ are chosen so $\left(z^{p}+1\right)^{1 / p}=z+O(1)$ near $\infty$. These model mappings commute with rotation by a $p$ th root of unity and are semiconjugate to $w \mapsto$ $\lambda_{n}^{p} w+1$ and $w \mapsto w+1$ under the substitution $w=z^{p}$. For $p>1$ the maps $T_{n}$ and $T$ are only defined near $z=\infty$.

Note that $f_{n} \rightarrow f$ is in the dominant normal form produced by Theorem 7.2 , except that the fixed-point has been moved from zero to infinity. Thus a corresponding result holds whenever $\left(f_{n}, c_{n}\right) \rightarrow(f, c)$ dominantly.

Proof of Theorem 8.1. Partition the sphere $\widehat{\mathbb{C}}$ into disks $B \sqcup D$, where $D=\{z:$ $|z|>R\}$ and $R \gg 0$ is chosen so $D$ is contained well within in the domain where $f$ is univalent. Then $f$ is nearly linear on $D$.

We claim $f \mid D$ can be extended to a map $F: \widehat{\mathbb{C}} \rightarrow \widehat{\mathbb{C}}$ such that the iterates of $F$ are uniformly quasiconformal, with dilatation

$$
K\left(F^{n}\right)=1+O\left(R^{-1}\right)
$$

for all $n$. Assuming this claim, $F$ is conjugate by a $1+O\left(R^{-1}\right)$-quasiconformal map to a Möbius transformation $T(z)$. (To achieve this conjugacy, one constructs an $F$-invariant Beltrami differential $\mu$ with $|\mu|=O\left(R^{-1}\right)$ from the full orbit under $F$ of the standard structure on the sphere, and applies the measurable Riemann mapping theorem; see [41, Theorem 9].) Since $F=f$ near $\infty, T(z)$ must be parabolic, so we may assume $T(z)=z+1$ and the proof is complete.

It remains to construct $F$. The extension can be done directly by hand, or analytically as follows. Let

$$
\rho_{D}=\frac{2 R|d z|}{|z|^{2}-R^{2}}
$$

denote the Poincaré metric on $D$, and let $S f(z) d z^{2}$ be the Schwarzian derivative of $f$, a quadratic differential analytic near $z=\infty$. From the behavior of $\rho_{D}$ with 
respect to $R$ it follows that

$$
\|S f\|_{D}=\sup _{D} \frac{|S f(z)|}{\rho_{D}^{2}(z)}=O\left(R^{-2}\right) .
$$

Now the Ahlfors-Weill extension (cf. [18, §5.4]) prolongs any such $f$ with small Schwarzian to a quasiconformal homeomorphism $F: \widehat{\mathbb{C}} \rightarrow \widehat{\mathbb{C}}$ with $K(F)=1+$ $O\left(\|S f\|_{D}\right)=1+O\left(R^{-2}\right)$.

We claim

$$
F(z)=z+1+O\left(R^{-1}\right)
$$

This bound holds for $z \in D$ by our assumptions on $f$. To see it on $B$, first assume $F(z)-1$ fixes $\pm R$. Since $K(F)=1+O\left(R^{-2}\right), F(z)-1$ moves points at most distance $O\left(R^{-2}\right)$ in the Poincaré metric $\rho(z)|d z|$ on $\mathbb{C}-\{-R, R\}$; since $\rho(z)=O(1 / R)$ on $B$, the estimate follows. If $F(z)-1$ does not fix $\pm R$, it still moves these points at most Euclidean distance $O\left(R^{-1}\right)$, so after composition with an affine map of size $O\left(R^{-1}\right)$ these points are fixed and again the estimate follows.

From (8.2) we have that for large $R$ and all $n, \operatorname{Re}\left(F^{n}(z)-z\right) \asymp n$. Since $\operatorname{Re} F^{n}(z) \in[-R, R]$ if $F^{n}(z) \in B$, we see any orbit of $F$ includes at most $O(R)$ points in $B$. Thus $K\left(F^{n}\right)=1+O\left(R \cdot R^{-2}\right)=1+O\left(R^{-1}\right)$ for all $n$, establishing (8.1) and completing the proof.
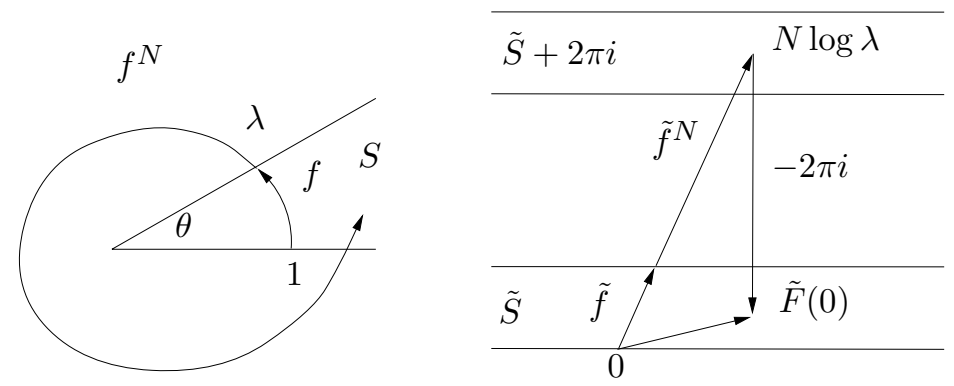

Figure 2. The first return map.

Renormalization. The analysis of nearly recurrent dynamics is facilitated by the renormalization or first return construction. This technique is central to the next proof, so we begin by explaining the idea in the linear case $f(z)=\lambda z$, with

$$
\log \lambda=L+i \theta
$$

$L>0$ and $0<\theta<\pi$ 
Consider the region

$$
S=\left\{z \in \mathbb{C}^{*}: 0 \leq \arg (z) \leq \theta\right\},
$$

and let $S / f$ denote the Riemann surface obtained from $S$ by gluing $z$ to $f(z)$ for $z \in \mathbb{R}_{+}$. The first return map $F: S \rightarrow S$ is defined by $F(z)=f^{N}(z)$ for the least $N>1$ with $f^{N}(z) \in S$. The first return occurs after the orbit of $z$ has moved once around the origin. The map $F$ descends to a biholomorphic map

$$
R f: S / f \rightarrow S / f
$$

called the renormalization of $f$.

Now the Riemann surface $S / f$ is a cylinder isomorphic to $\mathbb{C}^{*}$. Let us arrange this isomorphism so $0 \in \partial S$ corresponds to $0 \in \partial \mathbb{C}^{*}$; then $R f(z)=R(\lambda) \cdot z$ where

$$
\log R(\lambda)=\frac{4 \pi^{2}}{\log \lambda}
$$

To check this formula, it is useful to pass to the universal cover $\pi: \mathbb{C} \rightarrow \mathbb{C}^{*}$, where $\pi(z)=e^{z}$ and $\underset{S}{S}$ is covered by the strip $\tilde{S}=\{z: 0 \leq \operatorname{Im}(z) \leq \theta\}$ (Figure 2). Then $f$ lifts to $\tilde{f}(z)=z+\log \lambda$, and

$$
\tilde{F}(0)=\tilde{f}^{N}(0)-2 \pi i=N \log \lambda-2 \pi i
$$

for the least $N>1$ with $\tilde{f}^{N}(0) \in 2 \pi i+\tilde{S}$. The identification $\tilde{S} / \tilde{f} \cong \mathbb{C}^{*}$ is given by $\pi_{R}(z)=\exp (-2 \pi i z / \log \lambda)$, and thus

$$
R(\lambda)=(R f)(1)=\pi_{R}(\tilde{F}(0))
$$

which yields (8.3). In particular,

$$
\log |R(\lambda)|=\frac{4 \pi^{2}}{L+\theta^{2} / L}
$$

Since $\lambda_{n}=\exp \left(L_{n}+i \theta_{n}\right) \rightarrow 1$ horocyclically if and only if both $L_{n} \rightarrow 0$ and $\theta_{n}^{2} / L_{n} \rightarrow 0$, we have:

Proposition 8.4. For $\left|\lambda_{n}\right|>1$ we have $\lambda_{n} \rightarrow 1$ horocyclically iff $R\left(\lambda_{n}\right) \rightarrow \infty$.

Proof of Theorem 8.2. We apply the construction of Theorem 8.1 to each $f_{n}$. That is, writing $\widehat{\mathbb{C}}=D \cup B$ where $B=B(0, R)$ and $R \gg 0$, we apply the Ahlfors-Weill extension to $f_{n} \mid D$ obtain a quasiconformal mapping $F_{n}: \widehat{\mathbb{C}} \rightarrow \widehat{\mathbb{C}}$ with $F_{n}=f_{n}$ on 
$D, K\left(F_{n}\right)=1+O\left(R^{-2}\right)$ and

$$
F_{n}(z)=\lambda_{n} z+1+O\left(R^{-1}\right)
$$

for all $z \in \mathbb{C}$.

Our main task is to show that for $n$ and $R$ large, we have

$$
F_{n}^{i}(B) \cap B=\emptyset \text { for }|i|>3 R .
$$

This control on the recurrence of $B$ will imply $K\left(F_{n}^{i}\right)=1+O\left(R^{-1}\right)$ and thus $F_{n}$ is conjugate, with small dilatation, to an affine mapping. The proof of (8.5) breaks into 3 cases.

Case 1: $\lambda_{n}=1$. Then $\operatorname{Re}\left(F_{n}^{i}(z)\right)-\operatorname{Re} z \asymp i$ which implies (8.5).

Excluding the subsequence where Case 1 holds, we henceforth assume $\lambda_{n} \neq 1$ for all $n$. Then $f_{n}$ has a second fixed-point $a_{n}$ near the fixed-point of the affine map $\lambda_{n} z+1$; in fact

$$
a_{n}=\frac{1}{1-\lambda_{n}}+O(1)
$$

by Rouché's theorem, and

$$
\lambda_{n}^{\prime}=f^{\prime}\left(a_{n}\right)=\lambda_{n}+O\left(\left|1-\lambda_{n}\right|^{2}\right)
$$

since $f^{\prime}(z)=\lambda_{n}+O\left(1 / z^{2}\right)$. From this we find $\lambda_{n}^{\prime} \rightarrow 1$ horocyclically as well.

Since the fixed-points $\infty$ and $a_{n}$ behave the same, we can exchange them if necessary (by a Möbius conjugacy close to the identity) to arrange that $\left|\lambda_{n}\right|>1$ for all $n$. Then we can write

$$
\log \lambda_{n}=L_{n}+i \theta_{n} \in \mathbb{H}
$$

with $\theta_{n} \rightarrow 0$.

Case 2: Radial convergence. Suppose we have the radial convergence condition $\left|\theta_{n}\right|<M L_{n}$ for all $n$. Then for all $n \gg 0$ :

$$
\frac{\left|\lambda_{n}\right|-1}{\left|\lambda_{n}-1\right|} \sim \frac{L_{n}}{\left|L_{n}+i \theta_{n}\right|}>\frac{1}{M+1} .
$$

Let $b_{n}=\left(1-\lambda_{n}\right)^{-1}$ be the fixed-point of the linear map $\lambda_{n} z+1$. On a large scale, $F_{n}$ repels from $b_{n}$, and

$$
\left|F_{n}(z)-b_{n}\right|=\left|z-b_{n}\right|+\left(\left|\lambda_{n}\right|-1\right)\left|z-b_{n}\right|+O\left(R^{-1}\right) .
$$


Now $z \in B$ satisfies $\left|z-b_{n}\right|>\left|b_{n}\right| / 2(n \gg 0)$; setting $z_{i}=F_{n}^{i}(z)$ by induction we find

$$
\left|z_{i+1}-b_{n}\right|-\left|z_{i}-b_{n}\right| \geq \frac{\left|\lambda_{n}\right|-1}{2\left|\lambda_{n}-1\right|}-O\left(R^{-1}\right) \geq \frac{1}{2 M+3}
$$

when $R$ is sufficiently large (using (8.6)). Thus the distance $\left|z_{i}-b_{n}\right|$ increases linearly along a forward orbit starting in $B$, establishing (8.5) for $i>0$. For backward iteration, move the other fixed-point $a_{n}$ to infinity and repeat the argument.

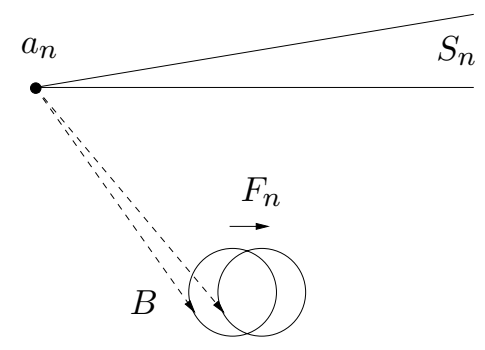

Figure 3. Horocyclic dynamics.

Case 3: Horocyclic convergence. For the last case we assume both $\theta_{n}^{2} / L_{n} \rightarrow 0$ (horocyclic convergence) and $\left|\theta_{n}\right|>L_{n}$ (since otherwise we have radial convergence). For convenience we also assume $\theta_{n}>0$.

Consider the measure of rotation

$$
\rho_{n}(z)=\frac{F_{n}(z)-a_{n}}{z-a_{n}}
$$

We claim

$$
\arg \rho_{n}(z) \asymp \theta_{n}
$$

for all $n$ sufficiently large.

First, for $z \in B$, the triangle with vertices $\left(z, F_{n}(z), a_{n}\right)$ has two long sides of length about $\theta_{n}^{-1}$ and a short side of length $\left|z-F_{n}(z)\right| \asymp 1$ nearly parallel to the real axis. The condition $\theta_{n}>L_{n}$ implies $a_{n}$ avoids a cone of definite angle around the real axis, so the angle $\arg \rho_{n}(z)$ between the long sides is comparable to $\theta_{n}$ (see Figure 3).

Now $\rho_{n}(z)$ is holomorphic on $\widehat{\mathbb{C}}-B$ (with $\rho_{n}(\infty)=\lambda_{n}$ and $\left.\rho_{n}\left(a_{n}\right)=\lambda_{n}^{\prime}\right)$; since (8.7) holds on $\partial B$, it holds throughout $\widehat{\mathbb{C}}-B$ by the maximum principle.

Because of (8.7) the orbits of $F_{n}$ circulate around $a_{n}$ and pass through the region $S_{n}$ bounded by a positive ray through $a_{n}$ and its image (Figure 3 again). Note that $F_{n}=f_{n}$ is holomorphic on $S_{n}$ and thus $S_{n} / f_{n}$ is naturally a Riemann 
surface isomorphic to $\mathbb{C}^{*}$. The first return construction determines a $1+O\left(R^{-1}\right)$ quasiconformal map

$$
R F_{n}: S_{n} / f_{n} \rightarrow S_{n} / f_{n}
$$

since any orbit starting in $B$ departs within $O(R)$ iterates and lands in $S_{n}$ before returning again. The orbits passing through $B$ are confined to a round annulus $A_{n} \subset S_{n} / f_{n}$ of modulus $O(R)$ and $R F_{n}$ is holomorphic elsewhere.

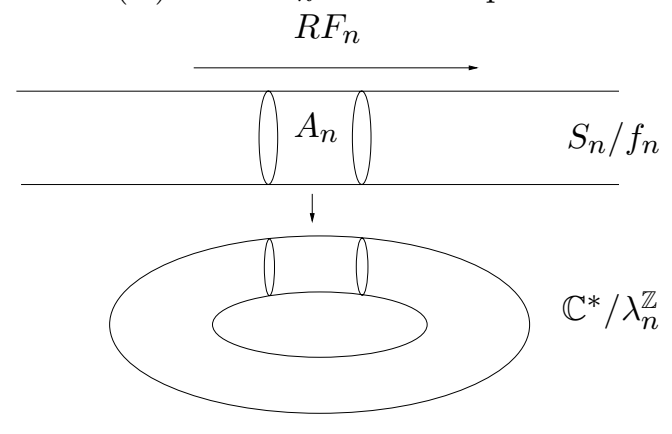

Figure 4. Cylinder and quotient torus.

Think of $S_{n} / f_{n} \cong \mathbb{C}^{*}$ as an infinite cylinder of unit radius. Then $A_{n}$ is a subcylinder of width $O(R)$. The map $R F_{n}$ is approximately an isometry of the whole cylinder, translating by distance

$$
\log \left|R F_{n}^{\prime}(\infty)\right|=\log \left|R\left(\lambda_{n}\right)\right| \rightarrow \infty
$$

(by Proposition 8.4). Thus for all $n \gg 0, A_{n}$ embeds in the quotient torus $\mathbb{C}^{*} / \lambda_{n}^{\mathbb{Z}}$ and

$$
R F_{n}^{i}\left(A_{n}\right) \cap A_{n}=\emptyset
$$

for $i \neq 0$ (see Figure 4). This means $B$ never returns to itself after circulating around $a_{n}$, so (8.5) holds in this final case as well.

Completion of the proof. To construct the linearizations, we now define $F_{n^{-}}$ invariant Beltrami differentials $\mu_{n}$ converging to an $F$-invariant $\mu$.

Observe that for any $z \in \widehat{\mathbb{C}}$, by we have $F_{n}^{i}(z) \in D$ for all $i \gg 0$. Indeed, for $z \in B$ we can take $i=3 R+1$. Let $\mu_{n}(z)$ be the complex dilatation of $F_{n}^{i}(z)$, $i \gg 0$; it is well-defined since $F_{n}$ is conformal on $D$. In other words, $\mu_{n}(z)$ is the pullback of the standard complex structure on $D$ along the orbit of $z$; it is clearly invariant. Define by the same procedure an $F$-invariant Beltrami differential $\mu$.

The Schwarzians of the maps $f_{n}$ satisfy $S f_{n} \rightarrow S f$ on $D$ in the $C^{\infty}$ topology, so their Ahlfors-Weill extensions satisfy $F_{n} \rightarrow F$ smoothly on $B$. Therefore $\mu_{n} \rightarrow \mu$ 
a.e. on $\widehat{\mathbb{C}}$ (using (8.5) to verify that $\mu_{n}(z)$ and $\mu(z)$ can be defined using $F_{n}^{i}$ and $F^{i}$ where $i$ depends only on $z$.)

Let $\phi_{n}: \widehat{\mathbb{C}} \rightarrow \widehat{\mathbb{C}}$ be the unique quasiconformal map with complex dilatation $\mu_{n}$, normalized to fix 0 and $\infty$ and with $\phi_{n}\left(F_{n}(0)\right)=1$. Let $\phi$ with dilatation $\mu$ be similarly normalized for $F$. Since $\mu_{n} \rightarrow \mu$, we have $\phi_{n} \rightarrow \phi$ uniformly on $\widehat{\mathbb{C}}$. Then $\phi_{n} \rightarrow \phi$ conjugates $F_{n} \rightarrow F$ to $T_{n} \rightarrow T$, where $T_{n}(z)=\alpha_{n} z+1$ and $T(z)=z+1$. Since $F_{n}=f_{n}$ and $F=f$ outside $B$, the proof is almost complete.

It remains only to replace $\alpha_{n}$ with $\lambda_{n}$. To this end, consider the complex tori forming the local quotient space for the dynamics of $f_{n}$ and $T_{n}$ near $z=\infty$. The map $\phi_{n}$ descends to a map

$$
\Phi_{n}: \mathbb{C}^{*} / \lambda_{n}^{\mathbb{Z}} \rightarrow \mathbb{C}^{*} / \alpha_{n}^{\mathbb{Z}}
$$

between these quotient tori, with $K\left(\Phi_{n}\right)=K\left(\phi_{n}\right)$. Since $\Phi_{n}$ is conformal outside the image of $A_{n}$, and $R\left(\lambda_{n}\right) \rightarrow \infty$, we see $\Phi_{n}$ is conformal on most of the torus $\mathbb{C}^{*} / \lambda_{n}^{\mathbb{Z}}$ (Figure 4). Thus the Teichmüller mapping $\Psi_{n}$ in the homotopy class of $\Phi_{n}$ has dilatation $K\left(\Psi_{n}\right) \rightarrow 1$. Replacing $\phi_{n}$ with $\psi_{n}^{-1} \circ \phi_{n}$ for suitable lifts of $\Psi_{n}$, we can replace $\alpha_{n}$ with $\lambda_{n}$ and complete the proof.

Proof of Theorem 8.3. For simplicity assume $\left|\lambda_{n}\right| \geq 1$. The mappings $f_{n}$ and $f$, like their models $T_{n}$ and $T$, are asymptotic to $p$-fold coverings of affine mappings. To see this, make the change of variables $w=z^{p}$; then

$$
\begin{aligned}
f(w) & =w+p+O\left(w^{-1 / p}\right), \\
f_{n}(w) & =\lambda_{n}^{p} w+p \lambda_{n}^{p-1}+O\left(w^{-1 / p}\right),
\end{aligned}
$$

where $f$ and $f_{n}$ are understood as multi-valued functions that become well-defined on a $p$-sheeted covering of the $w$-plane. Using the equations above, the dynamics can be analyzed in a manner similar to the preceding proof.

For example, under $f$ the point $z=\infty$ is a fixed-point of multiplicity $p+1$. It is attracting along the nearly invariant rays where $z^{p} \in \mathbb{R}_{+}$and repelling along the rays $z^{p} \in \mathbb{R}_{-}$. For $\lambda_{n} \neq 1$ this fixed-point splits into an attracting point at $z=\infty$ with multiplier $\lambda_{n}^{-1}$, and $p$ symmetrically arrayed repelling points $z \approx\left(1-\lambda_{n}\right)^{-1 / p}$ with multipliers $\approx \lambda_{n}^{p}$.

The idea of the proof is to construct a conjugacy between $f_{n}$ and $T_{n}$ on each of $p$ sectors where the behavior is like that of a $p$ th root of an affine map. See Figure 5 , where $z=\infty$ has been moved by an inversion to the center of the picture. We will use double indices to label these sectors and associated objects; $S_{n j}, 0 \leq j<p-1$, will be the sectors associated to $f_{n}$.

To define these sectors, consider a large radius $R$, and let $R_{j}=R e^{2 \pi i j / p}$. For $R$ large enough, $f_{n}^{i}\left(R_{j}\right) \rightarrow \infty$ for all $n$. Join these points to form a path 


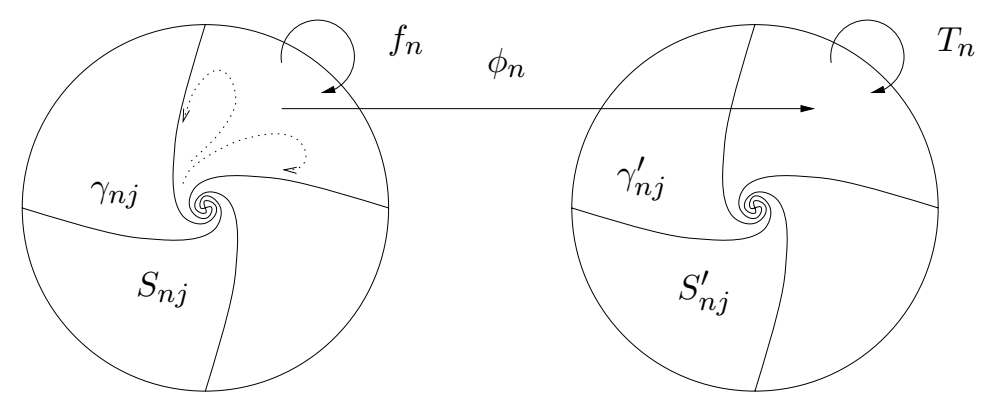

Figure 5. Multiple petals $(p=4)$.

$\gamma_{n j}:[0, \infty) \rightarrow \mathbb{C}$, defined by

$$
\gamma_{n j}(t)= \begin{cases}R_{j} & \text { if } t=0 \\ (1-t) R_{j}+t f_{n}\left(R_{j}\right) & \text { if } 0<t<1, \text { and } \\ f_{n}\left(\gamma_{n j}(t-1)\right) & \text { if } t \geq 1\end{cases}
$$

We can assume $\left|\lambda_{n}-1\right|$ is small, since otherwise $f_{n}$ and $T_{n}$ are conformally conjugate on a definite neighborhood of infinity. Then for $R$ large, each path $\gamma_{n j}$ is properly embedded and nearly straight in the region $R<|z|<2 R$. The paths divide

$$
D(R)=\{z:|z| \geq R\}
$$

into $p$ sectors $S_{n j}$ bounded by $\gamma_{n j}$ and $\gamma_{n, j+1}, j=0, \ldots, p-1$.

Next we glue together the edges of $S_{n j}$ to obtain a new dynamical system. That is, we identify $\gamma_{n j}(t)$ and $\gamma_{n, j+1}(t)$ for all $t$, to obtain from $S_{n j}$ a Riemann surface conformally equivalent to a punctured disk, and hence to $D\left(R^{p}\right)=\left\{z:|z| \geq R^{p}\right\}$. In fact there is a unique conformal map $\psi_{n j}: S_{n j} \rightarrow D\left(R^{p}\right)$ that identifies the parameterized edges, sends $\partial D(R)$ to $\partial D\left(R^{p}\right)$ and sends $R_{j}$ to $R^{p}$.

Conjugating $f_{n}$ by $\psi_{n j}$, we obtain a holomorphic map $f_{n j}$ defined near $\infty$ in $D\left(R^{p}\right)$ with

$$
f_{n j}(z)=\lambda_{n j} z+O(1)
$$

where $\lambda_{n j} \approx \lambda_{n}^{p}$. More precisely, for $R$ large enough we have

$$
d\left(\log \lambda_{n j}, \log \lambda_{n}^{p}\right) \leq \epsilon / 2
$$

in the hyperbolic metric on the upper half-plane, because $\psi_{n j}(z) \approx z^{p}$. 
Finally set $f_{\infty}=f$, and carry out the same construction with $n=\infty$. Then

$$
f_{\infty, j}(z)=z+c_{j}+O(1 / z)
$$

with $c_{j} \neq 0$. (In fact $c_{j} \approx p$ for $R$ large, because $\psi_{\infty, j}(z) \approx z^{p}$.) Then

$$
f_{n j} \rightarrow f_{\infty, j}
$$

on a neighborhood of $z=\infty$, and the corresponding multipliers $\lambda_{n j}$ converge to 1 horocyclically.

Now construct similar paths $\gamma_{n j}^{\prime}$ and sectors $S_{n j}^{\prime}$ for the model map $T_{n}$. Since $T_{n}$ commutes with rotation by a $p$ th root of unity, the edges of $S_{n j}^{\prime}$ can be glued together by a rotation, and the quotient map $\psi_{n j}: S_{n j}^{\prime} \rightarrow D\left(R^{p}\right)$ is given just by $\psi_{n j}(z)=z^{p}$. Therefore the quotient dynamics is given by

$$
T_{n j}(z)=\lambda_{n}^{p}(z+1) .
$$

By Theorem 8.2, there are $(1+\epsilon)$-quasiconformal maps $\phi_{n j} \rightarrow \phi_{j}$ defined near $\infty$ that conjugate $f_{n j} \rightarrow f_{\infty, j}$ to $T_{n j} \rightarrow T_{\infty, j}$. (Here we use an additional conjugacy controlled by (8.8) to replace $\lambda_{n j}$ with $\lambda_{n}^{p}$.)

Increasing $R$ again, we can assume $D\left(R^{p}\right)$ is contained in the domain and range of $\phi_{n j}$. Note that $T_{n j}(z)$ commutes with all rotations about $z=\left(1-\lambda_{n}^{p}\right)^{-1}$, or all translations if $\lambda_{n}=1$. Thus we can compose with a conformal automorphism of $T_{n j}$ to arrange that $\phi_{n j}\left(R^{p}\right)=R^{p}$ for all $n \gg 0$. We can also arrange that

$$
\phi_{n j} \circ \psi_{n j} \circ \gamma_{n j}(t)=\psi_{n j}^{\prime} \circ \gamma_{n j}^{\prime}(t)
$$

for all $t \geq 0$. Indeed, the paths above descends to nearly straight quasicircles on the quotient torus or cylinder for $T_{n j}$, passing through the same point (the image of $R^{p}$ ), so by a small quasiconformal isotopy they can be made to coincide. This isotopy lifts to an isotopy of $\phi_{n j}$ commuting with the dynamics and achieving (8.9).

By (8.9) it is evident that the lifts

$$
\tilde{\phi}_{n j}=\left(\psi_{n j}^{\prime}\right)^{-1} \circ \phi_{n j} \circ \psi_{n j}
$$

send $S_{n j}$ to $S_{n j}^{\prime}$ near $z=\infty$ and send $\gamma_{n j}$ to $\gamma_{n j}^{\prime}$ respecting parameterizations. Thus the lifts fit together to form a $(1+\epsilon)$-quasiconformal map $\phi_{n}$ conjugating $f_{n}$ to $T_{n}$ near $\infty$.

By the normalization $\phi_{n}(R)=R$ and the bound $K\left(\phi_{n}\right) \leq 1+\epsilon$ on the dilatation, the maps $\phi_{n}$ range in a compact family and hence $\phi_{n} \rightarrow \phi$ after passing to a subsequence. 


\section{Notes.}

1. A topological version of Theorem 8.1 was obtained by Camacho in [6].

2. See e.g. [47] for the analytic classification of parabolic fixed-points.

3. Parabolic bifurcations can be analyzed in great detail by the technique of Ecalle cylinders, implicit in the renormalization construction above. For more details and examples see [14], [13] and [38].

4. The renormalization construction is applied to small denominators in [48].

\section{Continuity of Julia sets}

The Julia set $J(f)$ determines a map

$$
J: \operatorname{Rat}_{d} \rightarrow \mathrm{Cl}(\widehat{\mathbb{C}})
$$

from the space of all rational maps of degree $d$ to the space of compact subsets of the sphere. Here Rat ${ }_{d}$ is given the algebraic topology and $\mathrm{Cl}(\widehat{\mathbb{C}})$ the Hausdorff topology (recalled below).

As is well-known, $J(f)$ varies discontinuously at many points $f \in \operatorname{Rat}_{d}$. For example, if $f$ has a Siegel disk with center $x$, then $x$ can be made repelling by a slight perturbation of $f$. We then obtain $f_{n} \rightarrow f$ with $x \in J\left(f_{n}\right)$ but $x \notin J(f)$. Parabolic implosions provide another source of discontinuity [13], [16]. In fact $J(f)$ varies continuously on a neighborhood of $f$ in Rat $_{d}$ iff $f$ is structurally stable. Conjecturally, $f$ is structurally stable iff $f$ is expanding. See [24, Thm. 4.2, Conj. 1.1] for more details.

In this section we give a condition that insures $J\left(f_{n}\right) \rightarrow J(f)$ when $f$ is geometrically finite. We will establish:

Theorem 9.1. (Continuity of $J$ ) Let $f$ be geometrically finite, and suppose $f_{n} \rightarrow$ $f$ horocyclically, preserving critical relations. Then $f_{n}$ is geometrically finite for all $n \gg 0$ and

$$
J\left(f_{n}\right) \rightarrow J(f)
$$

in the Hausdorff topology.

Algebraic limits. We say rational maps $f_{n}$ converge to $f$ algebraically if $\operatorname{deg} f_{n}=$ $\operatorname{deg} f$ and, when $f_{n}$ is expressed as the quotient of two polynomials, the coefficients can be chosen to converge to those of $f$. Equivalently, $f_{n} \rightarrow f$ uniformly in the spherical metric.

Given that $f_{n} \rightarrow f$ algebraically, we can further qualify the notion of convergence by imposing the following conditions. 
Critical relations. Let $b \in J(f)$ be a preperiodic critical point, satisfying $f^{i}(b)=$ $f^{j}(b)$ for some $i>j>0$. Suppose for all such $b$ and for all $n \gg 0$, the maps $f_{n}$ have critical points $b_{n} \in J\left(f_{n}\right)$ with the same multiplicity as $b, b_{n} \rightarrow b$ and $f_{n}^{i}\left(b_{n}\right)=f_{n}^{j}\left(b_{n}\right)$. Then we say $f_{n} \rightarrow f$ preserving critical relations.

Horocyclic and radial convergence of rational maps. It is a basic fact that a rational map $f$ has only a finite number of parabolic cycles [7, Thm. III.2.4]. Thus for a suitable $k>0$, every parabolic point $c$ of $f^{k}$ is a fixed-point with multiplier $\left(f^{k}\right)^{\prime}(c)=1$. We say $f_{n} \rightarrow f$ horocyclically (or radially) if for each such parabolic fixed-point $c$ of $f^{k}$, there are fixed-points $c_{n}$ of $f_{n}^{k}$ such that

(a) The pairs $\left(f_{n}^{k}, c_{n}\right) \rightarrow\left(f^{k}, c\right)$ dominantly in the space of maps with fixedpoints $\mathcal{F}$ introduced in $\S 8$; and

(b) The multipliers $\lambda_{n} \rightarrow 1$ horocyclically (or radially), where $\lambda_{n}=\left(f_{n}^{k}\right)^{\prime}\left(c_{n}\right)$.

The Hausdorff topology. Recall that compact sets $K_{n} \rightarrow K$ in the Hausdorff topology if:

(a) Every neighborhood of a point $x \in K$ meets all but finitely many $K_{n}$; and

(b) If every neighborhood of $x$ meets infinitely many $K_{n}$, then $x \in K$.

We define $\lim \inf K_{n}$ as the largest set satisfying (a), and $\lim \sup K_{n}$ as the smallest set satisfying (b) $[21, \S 2-16]$. Then $K_{n} \rightarrow K$ is equivalent to $\lim \sup K_{n}=$ $\liminf K_{n}=K$.

The next result is well-known (cf. $[13, \S 5])$ :

Lemma 9.2. If $f_{n} \rightarrow f$ algebraically, then $J(f) \subset \liminf J\left(f_{n}\right)$.

Proof. Any neighborhood of $x \in J(f)$ contains a repelling periodic point which persists nearby in $J\left(f_{n}\right)$ for all $n \gg 0$.

Here is the model result for showing that parabolic basins move continuously.

Lemma 9.3. Let $\lambda_{n} \rightarrow 1$ horocyclically, and let $T_{n}(z)=\lambda_{n} z+1$. Then for any $R>0$ there exists an $N$ such that

$$
\left|T_{n}^{k}(x)\right|>R
$$

whenever $|x|<R$ and $n,|k|>N$.

Proof. We treat the case where $x=0$; the case where $|x|$ is bounded by $R$ is similar. By horocyclic convergence we can write $\lambda_{n}=\exp \left(L_{n}+i \theta_{n}\right)$ with $L_{n} \rightarrow 0$ 
and $\theta_{n}^{2} / L_{n} \rightarrow 0$. Then

$$
T_{n}^{k}(0)=\lambda_{n}^{k-1}+\cdots+\lambda_{n}+1=\frac{\lambda_{n}^{k}-1}{\lambda_{n}-1} .
$$

For $n$ large enough, $\left|\lambda_{n}-1\right| \ll 1 / R$ so we need only worry about the case where the numerator is close to zero. In that case

$$
\left|T_{n}^{k}(0)\right| \asymp \frac{\left|k L_{n}+i\left\{k \theta_{n}\right\}\right|}{\left|L_{n}+i \theta_{n}\right|}
$$

where $\left\{k \theta_{n}\right\}=k \theta_{n}+2 \pi j$ and $j$ is an integer chosen to give the value closest to zero.

If $|k|<\left|1 / \theta_{n}\right|$ then $j=0$ and we have $\left|T_{n}^{k}(0)\right| \asymp|k|$, so we get $\left|T_{n}^{k}(0)\right|>R$ by taking the lower bound $N$ on $|k|$ large enough. If $|k| \geq\left|1 / \theta_{n}\right|$, then we have

$$
\frac{\left|k L_{n}+i\left\{k \theta_{n}\right\}\right|}{\left|L_{n}+i \theta_{n}\right|} \geq \frac{\left|L_{n} / \theta_{n}\right|}{\left|L_{n}+i \theta_{n}\right|}=\frac{1}{\left|\theta_{n}+i \theta_{n}^{2} / L_{n}\right|} \rightarrow \infty
$$

as $n \rightarrow \infty$ (by horocyclic convergence), so $\left|T_{n}^{k}(0)\right|>R$ in this case by taking the lower bound $N$ on $n$ sufficiently large.

Remark. The preceding result is related to the fact that the cyclic Kleinian groups $\Gamma_{n}=\left\langle T_{n}(z)=\lambda_{n} z+1\right\rangle$ converge geometrically to $\Gamma=\langle T(z)=z+1\rangle$; their quotient Riemann surfaces $\Omega\left(\Gamma_{n}\right) / \Gamma_{n}$ are complex tori converging to the infinite cylinder $\mathbb{C} / \Gamma \cong \mathbb{C}^{*}$. Compare $[28$, Thm. 5.1].

Proof of Theorem 9.1. (Continuity of $J$ ). The map $f$ has at most $2 \operatorname{deg}(f)-2$ attracting, superattracting or parabolic cycles. Thus by replacing $f_{n} \rightarrow f$ with $f_{n}^{k} \rightarrow f^{k}$ (which does not change the Julia sets), we can assume that all such cycles of $f$ are actually fixed points. We can also assume that $f^{\prime}(c)=1$ at each parabolic fixed-point $c$.

Since $f_{n} \rightarrow f$ algebraically, we have $J(f) \subset \liminf J\left(f_{n}\right)$. So to prove $J\left(f_{n}\right) \rightarrow$ $J(f)$, we need only show $\lim \sup J\left(f_{n}\right) \subset J(f)$. This amounts to showing, for each $x \in \Omega(f)=\widehat{\mathbb{C}}-J(f)$ (the Fatou set of $f$ ), there exists a neighborhood $U$ of $x$ such that $U \subset \Omega\left(f_{n}\right)$ for all $n \gg 0$.

Since the Fatou set is totally invariant, we can replace $x$ with a finite iterate $f^{i}(x)$ at any stage of the argument.

Because $f$ is geometrically finite, under iteration $f^{i}(x)$ converges to an attracting, superattracting or parabolic fixed-point $c$ of $f$.

Attracting and superattracting fixed-points. First suppose $c$ is attracting or superattracting. Then this behavior persists under algebraic perturbation of $f$. 
In fact there is a small neighborhood $U$ of $c$ such that $f_{n}(U) \subset U$ for all $n \gg 0$. Thus $U \subset \Omega\left(f_{n}\right)$. Choosing $i$ such that $f^{i}(x) \in U$, we have shown a neighborhood of $x$ persists in the Fatou set for large $n$.

Parabolics with one petal. Now suppose $c$ is a parabolic point with one petal. By our assumption of horocyclic convergence, there are fixed-points $c_{n}$ of $f_{n}$ such that $\left(f_{n}, c_{n}\right) \rightarrow(f, c)$ dominantly and the multipliers $\lambda_{n} \rightarrow 1$ horocyclically. By Theorem 7.2, we can first move $c_{n}$ to $\infty$, then make an analytic coordinate change (depending on $n$ ) near $\infty$ such that

$$
\begin{aligned}
f_{n}(z) & =\lambda_{n} z+1+O\left(z^{-1}\right), \\
f(z) & =z+1+O\left(z^{-1}\right) .
\end{aligned}
$$

Applying Theorem 8.2, we can make a further quasiconformal coordinate change near $\infty$ to arrive at the linearized dynamics

$$
\begin{aligned}
T_{n}(z) & =\lambda_{n} z+1, \\
T(z) & =z+1 .
\end{aligned}
$$

In summary, there is an $R$ such that for all $n \gg 0$, the linearized dynamics $T_{n}$ on the neighborhood $|z|>R$ of $\infty$ is topologically conjugate to the dynamics of $f_{n}$ on a neighborhood of $c_{n}$. The conjugacy $\phi_{n}$ from $f_{n}$ to $T_{n}$ converges to a conjugacy $\phi$ from $f$ to $T$.

Replacing $x$ with $f^{i}(x)$ for $i \gg 0$, we can assume that $x^{\prime}=\phi(x)$ is defined, and satisfies $\operatorname{Re} x^{\prime}>R$ (since the real part increases under iteration by $T$ ). We claim there is a neighborhood $V$ of $x^{\prime}$ and $N>0$ such that

$$
\left|T_{n}^{i}(z)\right|>R \text { for all } z \in V, i>0 \text { and } n>N .
$$

Indeed, by Lemma 9.3, we can choose $V$ and $N$ so (9.2) holds for $i>N$. But for $0<i \leq N$, we have $\left|T^{i}\left(x^{\prime}\right)\right|>R$; since $T_{n} \rightarrow T$, by further increasing $N$ we can obtain (9.2) for all $i>0$.

Since $\phi_{n} \rightarrow \phi$ and $\phi(x) \in V$, there is a neighborhood $U$ of $x$ such that $\phi_{n}(U) \subset$ $V$ for all $n \gg 0$. By (9.2), the iterates $f_{n}^{i}(z)$ then remain close to $c_{n}$ for all $z \in U$ and $i>0$. Thus $f_{n}^{i} \mid U$ is a normal family, so $U \subset \Omega\left(f_{n}\right)$ as desired.

Parabolics with multiple petals. The case of $p>1$ petals is very similar. Pass to any subsequence such that $J\left(f_{n}\right)$ converges in the Hausdorff topology. By Theorems 7.2 and 8.3, after passing to a further subsequence we obtain topological 
conjugacies $\phi_{n} \rightarrow \phi$ to the dynamical systems $T_{n} \rightarrow T$, where

$$
\begin{aligned}
T_{n}(z) & =\lambda_{n}\left(z^{p}+1\right)^{1 / p}, \\
T(z) & =\left(z^{p}+1\right)^{1 / p} .
\end{aligned}
$$

By composing with the map $z \mapsto z^{p}$, we obtain a semiconjugacy $\phi_{n} \rightarrow \phi$ to the linearized dynamics (9.1). Choose $V$ as before and $U$ with $\phi_{n}(U) \subset V$, we again find $U \subset \Omega\left(f_{n}\right)$ for all $n \gg 0$.

Therefore the original sequence satisfies $J\left(f_{n}\right) \rightarrow J(f)$.

Geometric finiteness. By algebraic convergence, any critical point $b_{n}$ of $f_{n}$ is close to a critical point $b$ of $f$. If $b \in J(f)$, then $b$ is preperiodic, and so is $b_{n}$ for all $n \gg 0$ by our assumption that critical point relations are preserved. If $b \in \Omega(f)$, then $b_{n} \in \Omega\left(f_{n}\right)$ for all $n \gg 0$, since $J\left(f_{n}\right) \rightarrow J(f)$. Thus for all $n \gg 0$, all critical points in $J\left(f_{n}\right)$ are preperiodic, so $f_{n}$ is geometrically finite.

\section{Parabolics and Poincaré series}

In this section we continue our study of parabolic bifurcations. We establish, under suitable conditions, uniform convergence of the Poincaré series. This uniformity controls the concentration of invariant densities as parabolics are created.

Poincaré series of germs. For $f \in \mathcal{G}$ we define the (forward) Poincaré series by

$$
P_{\delta}(f, x)=\sum_{i \geq 0}\left|\left(f^{i}\right)^{\prime}(x)\right|_{\sigma}^{\delta}
$$

where the derivative is measured in the spherical metric $\sigma$. To study the rate of convergence, we define for any open set $V$ the sub-sum

$$
P_{\delta}(f, V, x)=\sum_{f^{i}(x) \in V}\left|\left(f^{i}\right)^{\prime}(x)\right|_{\sigma}^{\delta}
$$

In both sums $i \geq 0$ ranges only over values such that $f^{i}(x)$ is defined, i.e. such that $f^{j}(x) \in U(f)$ for $0 \leq j<i$.

Now consider a sequence

$$
\left(f_{n}, c_{n}, \delta_{n}\right) \rightarrow(f, c, \delta)
$$

in $\mathcal{F} \times \mathbb{R}_{+}$such that

(a) $\left(f_{n}, c_{n}\right)$ converges to $(f, c)$ dominantly; and 
(b) $\delta>p /(p+1)$ if $(f, c)$ is parabolic with $p$ petals.

We say the Poincaré series for $\left(f_{n}, c_{n}, \delta_{n}\right)$ as above converge uniformly if, after suitably shrinking $U\left(f_{n}\right)$ and $U(f)$, for any compact set $K \subset U(f)-\{c\}$ and $\epsilon>0$, there exists a neighborhood $V$ of $c$ such that

$$
P_{\delta_{n}}\left(f_{n}, V, x\right)<\epsilon
$$

for all $n \gg 0$ and all $x \in K$. This means the tail of the series can be made small, independent of $n$, by choosing $V$ small enough.

Here is a simple case:

Theorem 10.1. Let $(f, c)$ be an attracting or superattracting fixed-point. Then the Poincaré series converge uniformly for any sequence $\left(f_{n}, c_{n}, \delta_{n}\right) \rightarrow(f, c, \delta)$.

Proof. After suitable restrictions, we can assume all points in $U\left(f_{n}\right)$ are attracted to $c_{n}$ under iteration of $f_{n}$, and $\left|f_{n}^{\prime}\right|<\lambda<1$ for all $n \gg 0$. Then the Poincaré series is dominated by the tail of a geometric series, namely $\sum_{f_{n}^{i}(x) \in V} \lambda^{i}$, and this bound is small when $V$ is a small neighborhood of $c$.

The main result of this section treats the parabolic case.

Theorem 10.2. Let $(f, c)$ be parabolic with $p$ petals and let $\lambda_{n}=f_{n}^{\prime}\left(c_{n}\right)$. If

(a) $\lambda_{n} \rightarrow 1$ radially; or

(b) $\lambda_{n} \rightarrow 1$ horocyclically, and $\delta>2 p /(p+1)$, then the Poincaré series for $\left(f_{n}, c_{n}, \delta_{n}\right)$ converge uniformly.

Proof. First consider the case where $\left(f_{n}, c_{n}\right)=\left(T_{n}, \infty\right)$ and $(f, c)=(T, \infty)$ are the model mappings

$$
\begin{aligned}
T_{n}(z) & =\lambda_{n}\left(z^{p}+1\right)^{1 / p}, \\
T(z) & =\left(z^{p}+1\right)^{1 / p},
\end{aligned}
$$

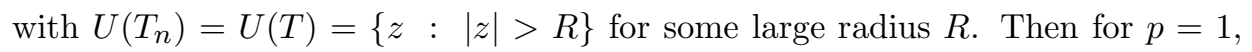
uniform convergence is proved in [28]. Indeed, $T_{n}$ and $T$ generate cyclic Kleinian groups with $L_{n} \rightarrow L$ geometrically by horocyclic convergence of $\lambda_{n} \rightarrow 1$. Uniform convergence of the Poincaré series under condition (a) or (b) then follows, from [28, Thms. 5.1 and $6.1-6.3]$.

For the case $p>1$, use the substitution $w=z^{p}$ to semiconjugate $T_{n} \rightarrow T$ to $S_{n} \rightarrow S$, where

$$
\begin{aligned}
S_{n}(w) & =\lambda_{n}^{p}(w+1), \\
S(w) & =w+1 .
\end{aligned}
$$


Then the spherical metric

$$
\sigma=\frac{2|d z|}{1+|z|^{2}}
$$

goes over to

$$
\rho=\frac{2|d w|}{p\left(|w|^{1+1 / p}+|w|^{1-1 / p}\right)} \asymp \frac{|d w|}{|w|^{1+1 / p}}
$$

for large $|w|$ (as in Proposition 3.2). To obtain uniform convergence, one simply repeats the proofs of $[28, \S 6]$ for this new metric. For example, bounds of the form

$$
P_{\delta_{n}}\left(T_{n}, V, x\right)=O\left(\sum_{k>K} k^{-2 \delta_{n}}\right)
$$

for $p=1$ become, for $p>1$,

$$
P_{\delta_{n}}\left(T_{n}, V, x\right)=O\left(\sum_{k>K} k^{-(1+1 / p) \delta_{n}}\right),
$$

and this is small for all $n, K \gg 0$ because $(1+1 / p) \delta_{n} \rightarrow(1+1 / p) \delta>1$.

Now consider the case of general $\left(f_{n}, c_{n}, \delta_{n}\right)$. Choose $L>1$ such that $\alpha_{n}=$ $\delta_{n} / L>p /(p+1)$ for all $n \gg 0$. By Theorems 7.2 and 8.3, there are $L$-quasiconformal conjugacies $\phi_{n}, \phi$ sending suitable restrictions of $\left(f_{n}, c_{n}\right) \rightarrow(f, c)$ to the model mappings $\left(T_{n}, \infty\right) \rightarrow(T, \infty)$ of $(10.1)$. Restricting the domains sufficiently, we may assume $f_{n}, f, T_{n}$ and $T$ are univalent.

An $L$-quasiconformal map is Hölder continuous of exponent $1 / L$; in particular,

$$
\operatorname{diam}(B)=O\left(\operatorname{diam}\left(\phi_{n}(B)\right)^{1 / L}\right)
$$

for any ball $B \subset U(f)$ [2, Ch. III.C]. To conclude the proof, we will use this Hölder continuity to transport uniform convergence from $T_{n}$ to $f_{n}$.

Consider a fixed compact set $K \subset U(f)-\{c\}$ and $\epsilon>0$. Choose a compact set $K^{\prime} \subset U(T)-\{\infty\}$ such that $\phi_{n}(K) \subset K^{\prime}$ for all $n \gg 0$. By uniform convergence of the Poincaré series for $\left(T_{n}, \infty, \alpha_{n}\right)$, there is a neighborhood $V^{\prime}$ of $\infty$ such that

$$
\sup _{x \in K^{\prime}} P_{\alpha_{n}}\left(T_{n}, x, V^{\prime}\right)<\epsilon
$$

for all $n \gg 0$. Choose a neighborhood $V$ of $c$ such that $\phi_{n}(V) \subset V^{\prime}$ for all $n \gg 0$.

Now consider $x \in K$. For a small ball $B$ about $x$, the Koebe distortion theorem 
gives

$$
P_{\delta_{n}}\left(f_{n}, x, V\right) \asymp \sum_{f_{n}^{i}(x) \in V}\left(\operatorname{diam} f_{n}^{i}(B)\right)^{\delta_{n}} .
$$

Set $x_{n}=\phi_{n}(x)$ and $B_{n}=\phi_{n}(B)$. Then (10.2) yields

$$
\operatorname{diam} f_{n}^{i}(B)=O\left(\left(\operatorname{diam} \phi_{n}\left(f_{n}^{i}(B)\right)\right)^{1 / L}\right)=O\left(\left(\operatorname{diam} T_{n}^{i}\left(B_{n}\right)\right)^{1 / L}\right),
$$

and since $\delta_{n} / L=\alpha_{n}$, we have

$$
P_{\delta_{n}}\left(f_{n}, x, V\right)=O\left(\sum_{T_{n}^{i}\left(x_{n}\right) \in V^{\prime}}\left(\operatorname{diam} T_{n}^{i}\left(B_{n}\right)\right)^{\alpha_{n}}\right) .
$$

But Koebe again gives

$$
\sum_{T_{n}^{i}\left(x_{n}\right) \in V^{\prime}}\left(\operatorname{diam} T_{n}^{i}\left(B_{n}\right)\right)^{\alpha_{n}} \asymp P_{\alpha_{n}}\left(T_{n}, x_{n}, V^{\prime}\right)<\epsilon
$$

by our choice of $V^{\prime}$. Thus $P_{\delta_{n}}\left(f_{n}, x, V\right)=O(\epsilon)$ and we have uniform convergence of the Poincaré series.

\section{Continuity of Hausdorff dimension}

In this section we establish conditions for continuity of the Hausdorff dimension of the Julia set.

The continuity of dimension will generally come along with a package of additional properties. For economy of language, we say $f_{n} \rightarrow f$ dynamically if:

D1. $f_{n} \rightarrow f$ algebraically;

D2. The Julia sets satisfy $J\left(f_{n}\right) \rightarrow J(f)$ in the Hausdorff topology;

D3. H. $\operatorname{dim} J\left(f_{n}\right) \rightarrow$ H. $\operatorname{dim} J(f)$;

D4. The critical dimension satisfies $\alpha\left(f_{n}\right) \rightarrow \alpha(f)$;

D5. The maps $f_{n}$ and $f$ are geometrically finite for all $n \gg 0$; and

D6. The normalized canonical densities on $J\left(f_{n}\right)$ and $J(f)$ satisfy $\mu_{n} \rightarrow \mu$ in the weak topology on measures.

The terminology is meant to suggest that the dynamical and statistical features of $f_{n}$ (as reflected in its Julia set and invariant density) converge to those of $f$.

Here is the prototypical example: 
Theorem 11.1. If $f_{n} \rightarrow f$ algebraically and $f$ is expanding, then $f_{n} \rightarrow f$ dynamically.

Proof. Since expanding maps are open in the space of all rational maps, $f_{n}$ is expanding for all $n \gg 0$, and hence geometrically finite. By Theorem 9.1, $J\left(f_{n}\right) \rightarrow$ $J(f)$ in the Hausdorff topology.

Now the Julia sets of the expanding maps $f$ and $f_{n}, n \gg 0$, carry unique normalized invariant densities $\mu$ and $\mu_{n}$, by Corollary 6.7. The density $\mu_{n}$ has dimension $\alpha\left(f_{n}\right)$. But any weak accumulation point $\nu$ of $\mu_{n}$ gives an $f$-invariant density supported on $J(f)$, by convergence of Julia sets. Thus $\nu=\mu, \mu_{n} \rightarrow \mu$, and $\alpha\left(f_{n}\right) \rightarrow \alpha(f)$, the dimension of $\mu$. Since $\alpha(f)=\mathrm{H}$. $\operatorname{dim} J(f)$ for any geometrically finite rational map (Theorem 6.1), we have H. $\operatorname{dim} J\left(f_{n}\right) \rightarrow$ H. $\operatorname{dim} J(f)$, and thus $f_{n} \rightarrow f$ dynamically.

Our goal in this section is to obtain dynamic convergence in the presence of parabolic points and critical points in the Julia set. We will establish:

Theorem 11.2. (Dynamic convergence) Let $f$ be geometrically finite and let $f_{n} \rightarrow$ $f$ algebraically, preserving critical relations. Suppose:

(a) $f_{n} \rightarrow f$ radially; or

(b) $f_{n} \rightarrow f$ horocyclically, and

$$
\liminf \text { H. } \operatorname{dim} J\left(f_{n}\right)>\frac{2 p(f)}{p(f)+1} .
$$

Then $f_{n} \rightarrow f$ dynamically.

Recall $p(f)$ denotes the petal number of $f(\S 3)$. Condition (b) can be replaced by:

$\left(\mathrm{b}^{\prime}\right) f_{n} \rightarrow f$ horocyclically, and H. $\operatorname{dim} J(f)>2 p(f) /(p(f)+1)$,

since Theorems 6.1, 9.1 and Proposition 11.3 below imply

$$
\liminf \alpha\left(f_{n}\right)=\liminf \mathrm{H} \cdot \operatorname{dim} J\left(f_{n}\right) \geq \alpha(f)=\mathrm{H} \cdot \operatorname{dim} J(f) .
$$

Semicontinuity of dimension. Before proceeding to the proof, we remark that one inequality for the critical dimension is general and immediate:

Proposition 11.3. If $f_{n} \rightarrow f$ algebraically, then $\alpha(f) \leq \liminf \alpha\left(f_{n}\right)$.

Proof 1. Let $\alpha_{0}=\liminf \alpha\left(f_{n}\right)$. Pass to a subsequence such that $\alpha\left(f_{n}\right) \rightarrow \alpha_{0}$, and such that normalized $f_{n}$-invariant densities $\mu_{n}$ of dimension $\alpha\left(f_{n}\right)$ converge 
to weakly to a measure $\mu$. Then $\mu$ is an $f$-invariant density on $\widehat{\mathbb{C}}$, so by Corollary 4.5 its dimension $\alpha_{0}$ is an upper bound for $\alpha(f)$.

Proof 2. (Compare $[38, \S 1]$.) By Theorem 2.1 we have hyp- $\operatorname{dim}(f)=\alpha(f)$ for any rational map $f$. By structural stability, any hyperbolic set $X$ for $f$ gives rise to a nearby hyperbolic set $X_{n}$ for $f_{n}, n \gg 0$, admitting a conjugacy to $f \mid X$ with Hölder exponent tending to one. Thus H. $\operatorname{dim} X_{n} \rightarrow$ H. $\operatorname{dim} X$, so hyp-dim $(f) \leq$ $\lim \inf \operatorname{hyp}-\operatorname{dim}\left(f_{n}\right)$.

Corollary 11.4. Let $f$ be geometrically finite with $J(f)=\widehat{\mathbb{C}}$. If $f_{n} \rightarrow f$ algebraically, then $\mathrm{H} . \operatorname{dim} J\left(f_{n}\right) \rightarrow 2$.

Proof. By Theorems 2.1, 6.1 and the preceding result we have

$$
\begin{aligned}
\liminf \text { H. } \operatorname{dim} J\left(f_{n}\right) & \geq \liminf \text { H. } \operatorname{dim} J_{\operatorname{rad}}\left(f_{n}\right)=\liminf \alpha\left(f_{n}\right) \\
& \geq \alpha(f)=\text { H. } \operatorname{dim} J(f)=2 .
\end{aligned}
$$

Thus the main concern in proving continuity of dimension is to show H. $\operatorname{dim} J(f)$ is not too small. To do this, we show $J(f)$ supports a limiting density without atoms.

Proof of Theorem 11.2. (Dynamic convergence). By Theorem 9.1, we have $J\left(f_{n}\right) \rightarrow$ $J(f)$ and $f_{n}$ is geometrically finite for all $n \gg 0$. Let $\mu_{n}$ be the canonical normalized invariant density on $J\left(f_{n}\right)$; its dimension is $\delta_{n}=\alpha\left(f_{n}\right)$. Consider any subsequence such that $\mu_{n} \rightarrow \nu$ in the weak topology on measures, and $\delta_{n} \rightarrow \delta$. We will show $\nu=\mu$, the canonical density for $f$ of dimension $\alpha(f)$. This will complete the proof of dynamic convergence, since it implies $\alpha\left(f_{n}\right) \rightarrow \alpha(f)$ and thus H. $\operatorname{dim} J\left(f_{n}\right) \rightarrow$ H. $\operatorname{dim} J(f)$ by Theorem 6.1 .

Now $\nu$ is an $f$-invariant density of dimension $\delta$, supported on $J(f)$ by convergence of Julia sets. To prove $\mu=\nu$, it suffices by Corollary 6.6 to show $\nu$ has no atom at any preperiodic point $c \in J(f)$. To this end we will show for any $\epsilon>0$ there is a neighborhood $V$ of $c$ such that $\mu_{n}(V)<\epsilon$ for all $n \gg 0$.

Repelling points. We first illustrate the method of proof when $c$ is a repelling periodic point. Replacing $f$ with an iterate of $f$, we can assume $f(c)=c$. Then there are repelling fixed-points $c_{n} \rightarrow c$ for $f_{n}, n \gg 0$, and we can locally invert $f_{n}$ and $f$ to obtain a convergent sequence of attracting fixed-points $\left(g_{n}, c_{n}\right) \rightarrow(g, c)$ in $\mathcal{F}$. By Theorem 10.1, after suitably restricting $g_{n}$ and $g$ the Poincaré series for $\left(g_{n}, c_{n}, \delta_{n}\right)$ converge uniformly.

Choose an fundamental annulus $K \subset U(g)-\{c\}$, within the domain of linearization, such that $\{c\} \cup \bigcup_{0}^{\infty} g^{i}(K)$ covers a neighborhood $V$ of $c$. Enlarging 
$K$ slightly, we can also assume that

$$
V \subset\left\{c_{n}\right\} \cup \bigcup_{0}^{\infty} g_{n}^{i}(K)
$$

for all $n \gg 0$.

By uniform convergence of the Poincaré series, after shrinking $V$ we can assume $P_{\delta_{n}}\left(g_{n}, V, x\right)<\epsilon$ for all $x \in K$. Since $\mu_{n}$ has no atoms, $\nu_{n}\left(c_{n}\right)=0$ and we find

$$
\begin{aligned}
\mu_{n}(V) \leq \sum_{0}^{\infty} \mu_{n}\left(g_{n}^{i}(K) \cap V\right)= & \int_{K} \sum_{g_{n}^{i}(x) \in V}\left|\left(g_{n}^{i}\right)^{\prime}(x)\right|^{\delta_{n}} d \mu_{n}(x) \\
& =\int_{K} P_{\delta_{n}}\left(g_{n}, V, x\right) d \mu_{n}(x)<\epsilon \mu_{n}(K) \leq \epsilon
\end{aligned}
$$

for all $n \gg 0$. Since $\epsilon$ was arbitrary, $\nu$ has no atom at $c$.

Parabolic points. Now suppose $c$ is a parabolic point with $p$ petals. Replacing $f$ with an iterate we can assume $f(c)=c$ and $f^{\prime}(c)=1$. By assumption there are fixed-points $c_{n}$ of $f_{n}$ such that $\left(f_{n}, c_{n}\right) \rightarrow(f, c)$ dominantly. Under assumption (b) we also have

$$
\delta \geq \liminf \alpha\left(f_{n}\right)>2 p(f) /(p(f)+1) \geq 2 p /(p+1) .
$$

Locally inverting $f_{n} \rightarrow f$ as before we obtain, by Theorem 10.2, a sequence $\left(g_{n}, c_{n}, \delta_{n}\right) \rightarrow(g, c, \delta)$ with uniformly convergent Poincaré series.

Now on a small neighborhood of $c$, the maps $\left(f_{n}, c_{n}\right) \rightarrow(f, c)$ are topologically conjugate to the model maps $\left(T_{n}, \infty\right) \rightarrow(T, \infty)$ of Theorem 8.3. For the models it is clear that under iteration, for any $x$ in a small neighborhood $U$ of $\infty$, either

(a) $x$ is a repelling fixed-point; or

(b) the forward orbit of $x$ escapes from $U$; or

(c) the forward orbit of $x$ stays in $U$ and converges to an attracting or parabolic fixed-point.

Thus the same holds true for iteration of $f_{n}$ and $f$ on $x$ in a small enough neighborhood $V$ of $c$.

Now for $x \in V \cap J\left(f_{n}\right)$, only (a) and (b) are possible. Choosing $V$ small enough, we can find a compact annulus $K \subset U(g)-\{c\}$ such that any forward orbit of $f$ of type (b) must pass through $K$. Enlarging $K$ slightly, we can assume the same 
is true for $f_{n}, n \gg 0$. Then we have

$$
V \cap J\left(f_{n}\right) \subset R_{n} \cup \bigcup_{0}^{\infty} g_{n}^{i}(K)
$$

where $R_{n}$ is the set of fixed-points of $f_{n}$. Shrinking $V$ further, we can assume $P_{\delta_{n}}\left(f_{n}, V, x\right)<\epsilon$ for $x \in K$. Since $\mu_{n}$ has no atoms and is supported on $J\left(f_{n}\right)$, we have

$$
\mu_{n}\left(V \cap J\left(f_{n}\right)\right) \leq \sum \mu_{n}\left(V \cap g_{n}^{i}(K)\right)
$$

and we again conclude $\mu_{n}(V)<\epsilon$ for all $n \gg 0$ by (11.1).

Preperiodic points. Finally we treat the case of a preperiodic point $b \in J(f)$. Replacing $f$ with an iterate, we can assume $f(b)=c$ and $c$ is a fixed-point as above. Then we can lift the dynamics of $f$ near $c$ to a map $g=f^{-1} \circ f \circ f$ defined near $b$. From the fixed-points $c_{n} \rightarrow c$ for $f_{n}$ above, we obtain $b_{n} \rightarrow b$ with $f_{n}\left(b_{n}\right)=c_{n}$.

If $b$ is a critical point, where $f$ is locally of degree $d$, then the same is true for $b_{n}$ by our assumption that critical relations are preserved. Thus we can form $g_{n}=f_{n}^{-1} \circ f_{n} \circ f_{n}$ and obtain a sequence

$$
\left(g_{n}, b_{n}\right) \rightarrow(g, b)
$$

in $\mathcal{F}$.

Since $\left(f_{n}, c_{n}\right) \rightarrow(f, c)$ dominantly, $\left(g_{n}, b_{n}\right) \rightarrow(g, b)$ dominantly, by Proposition 7.4. We have $g_{n}^{\prime}\left(b_{n}\right)^{d}=f_{n}^{\prime}\left(c_{n}\right)$, so $g_{n}^{\prime}\left(b_{n}\right) \rightarrow g^{\prime}(b)$ radially (or horocyclically) under assumption (a) (or (b)) of the Theorem. In the case of horocyclic convergence, we also have $\delta>2 p(f) /(p(f)+1) \geq 2 p /(p+1)$ where $p$ is the petal number of $(g, b)$. Thus Theorems 10.1 or 10.2 the Poincaré series for $\left(g_{n}, b_{n}, \delta_{n}\right)$ converge uniformly. Since the density $\mu_{n}$ is $g_{n}$-invariant, we obtain as above a neighborhood $V$ of $b$ with $\mu_{n}(V)<\epsilon$ for all $n \gg 0$.

\section{Julia sets of dimension near two}

In this section we show parabolic bifurcations lead to rational maps with H. $\operatorname{dim} J\left(f_{n}\right) \rightarrow 2$.

The $p$-fold cover of a rank-two cusp. We begin by sketching the connection between parabolic points, rank-two cusps, geometric limits and Julia sets with $\operatorname{dim} J(f) \approx 2$. 
For $0<r<1 / 2$, consider the Hecke group $\Gamma_{r} \subset \operatorname{Isom}(\mathbb{H})$ generated by $p(z)=$ $z+1$ and $r(z)=-r^{2} / z$. The limit set $\Lambda_{r}$ of $\Gamma_{r}$ is a Cantor set contained in $\mathbb{R} \cup\{\infty\}$.

As $r \rightarrow 0, \Lambda_{r}$ squeezes down to the integers $\mathbb{Z} \cup\{\infty\}$. Nevertheless the lower bound

$$
\text { H. } \operatorname{dim} \Lambda_{r}>1 / 2
$$

holds for all $r>0$.

The lower bound (12.1) comes from the parabolic subgroup in $\Gamma_{r}$ generated by $p(z)=z+1$. To see it, recall that $\Lambda_{r}$ carries an invariant conformal density $\mu$ of dimension $\delta=\mathrm{H}$. $\operatorname{dim} \Lambda_{r}$ (cf. [28] and references therein). Let $\Lambda_{r}(n)$ be the part of the limit set closest to the integer $n$. Working in the spherical metric $\sigma=2|d z| /\left(1+|z|^{2}\right)$, we have

$$
\begin{aligned}
\mu\left(\Lambda_{r}\right) & =\mu\left(\bigcup_{-\infty}^{\infty} \Lambda_{r}(n)\right)=\sum_{n} \mu\left(p^{n}\left(\Lambda_{r}(0)\right)\right) \asymp \mu\left(\Lambda_{r}(0)\right) \sum_{n}\left|\left(p^{n}\right)^{\prime}(0)\right|_{\sigma}^{\delta} \\
& \asymp \sum_{n}\left|\frac{\left|\left(p^{n}\right)^{\prime}(0)\right|}{1+\left|p^{n}(0)\right|^{2}}\right|^{\delta}=\sum_{n} \frac{1}{\left(1+n^{2}\right)^{\delta}} ;
\end{aligned}
$$

since $\mu\left(\Lambda_{r}\right)$ is finite, we have $\delta>1 / 2$.

By the same reasoning, H. $\operatorname{dim} \Lambda(\Gamma)>1$ whenever a Kleinian group $\Gamma$ has a rank-two cusp.

Now let $f(z)$ be a rational map such that $z=\infty$ is a parabolic fixed-point with $p$ petals. We have seen in $\S 8$ that $f$ behaves like the model map

$$
T(z)=\left(z^{p}+1\right)^{1 / p},
$$

i.e. like a $p$-fold covering of a rank-one cusp. The parabolic behavior near $\infty$ gives the lower bound

$$
\text { H. } \operatorname{dim} J(f)>\frac{p}{p+1}
$$

as in Theorem 3.1. As the number of petals tends to infinity, this lower bound tends to one.

To obtain Julia sets with dimension near two, we consider rational maps $f_{n} \rightarrow f$ horocyclically such that the geometric limit of the dynamics contains a second transformation $g(z)$, defined near $z=\infty$, commuting with $f$ and behaving like

$$
S(z)=\left(z^{p}+\tau\right)^{1 / p},
$$


$\tau \in \mathbb{H}$. The dynamical system generated by the pair $\langle S, T\rangle$ is a $p$-fold cover of a rank-two cusp, and thus its critical exponent satisfies

$$
\delta(S, T) \geq \frac{2 p}{p+1}
$$

A limit of the conformal densities $\mu_{n}$ for $f_{n}$ gives a density $\mu$ invariant under $\langle f, g\rangle$, and we conclude that

$$
\liminf \text { H. } \operatorname{dim} J\left(f_{n}\right) \geq \frac{2 p}{p+1}-\epsilon
$$

The $\epsilon$ arises because $\langle g, f\rangle$ is only $(1+\epsilon)$-quasiconformally conjugate to $\langle S, T\rangle$.

Statement of the theorem. We now proceed to a formal treatment. Let $c$ be a fixed-point of a rational map $f$ with $f^{\prime}(c)=1$. Let $f_{n} \rightarrow f$ algebraically, with fixed-points $c_{n} \rightarrow c$. Let

$$
\lambda_{n}=\exp \left(L_{n}+i \theta_{n}\right)=f_{n}^{\prime}\left(c_{n}\right) \rightarrow 1
$$

with $\theta_{n} \rightarrow 0$. We say $\lambda_{n} \rightarrow 1$ along the $\eta$-horocycle, if

$$
\theta_{n}^{2} / L_{n} \rightarrow \eta \neq 0
$$

as $n \rightarrow \infty$. We allow both $\eta>0$ and $\eta<0$ (the fixed-points $c_{n}$ can be attracting or repelling, but not indifferent.)

Theorem 12.1. (Dimension along horocycles) Suppose $f$ has p petals at $c,\left(f_{n}, c_{n}\right)$ $\rightarrow(f, c)$ dominantly, and $\lambda_{n} \rightarrow 1$ along the $\eta$-horocycle. Then

$$
\liminf \alpha\left(f_{n}\right) \geq \frac{2 p}{p+1}-\epsilon
$$

where $\epsilon=\epsilon(\eta, f) \rightarrow 0$ as $\eta \rightarrow 0$.

Since $\alpha\left(f_{n}\right) \leq \mathrm{H} \cdot \operatorname{dim} J\left(f_{n}\right)$ (Theorem 2.1), the lower bound above also holds for $\lim \inf \mathrm{H} . \operatorname{dim} J\left(f_{n}\right)$. When the number of petals $p$ is large and $\eta$ is small, one finds H. $\operatorname{dim} J\left(f_{n}\right)$ is close to 2 for $n \gg 0$. Let

To begin the proof, we study geometric limits of the model mappings of $\S 8$.

$$
\begin{aligned}
T_{n}(z) & =\lambda_{n}\left(z^{p}+1\right)^{1 / p}, \\
T(z) & =\left(z^{p}+1\right)^{1 / p}
\end{aligned}
$$


be germs in $\mathcal{G}$ with $U\left(T_{n}\right)=U(T)=\{z:|z|>R\}$ for some $R \gg 0$.

Proposition 12.2. If $\lambda_{n} \rightarrow 1$ along the $\eta$-horocycle, then there is a subsequence of $n$ such that $T_{n}^{k(n)} \rightarrow S$, where

$$
S(z)=\left(z^{p}+\tau\right)^{1 / p}
$$

is defined on a neighborhood of $z=\infty, k(n)=\left[2 \pi / \theta_{n}\right]$ and $\operatorname{Im} \tau=-2 \pi p \eta$.

Proof. Consider the case $p=1$. Then

$$
T_{n}^{k}(z)=\lambda_{n}^{k} z+\frac{\lambda_{n}^{k}-1}{\lambda_{n}-1}
$$

Now $k(n)$ is chosen so that $\lambda_{n}^{k(n)} \rightarrow 1$, so the first term above converges. Noting that $\lambda_{n}-1 \sim i \theta_{n}$, we have

$$
\frac{\lambda_{n}^{k(n)}-1}{\lambda_{n}-1} \sim \frac{k(n) L_{n}+i\left(k(n) \theta_{n}-2 \pi\right)}{i \theta_{n}}=-i\left[\frac{2 \pi}{\theta_{n}}\right] \frac{L_{n}}{\theta_{n}}+\left(k(n)-\frac{2 \pi}{\theta_{n}}\right) .
$$

The imaginary term tends to $-2 \pi i \eta$, and the real term is $O(1)$, so after passing to a subsequence the quotient above converges and $T_{n}^{k(n)} \rightarrow S(z)=z+\tau$ with $\tau$ as above.

For the case $p>1$, write $T_{n}(z)=\left(\lambda_{n}^{p} z^{p}+1\right)^{1 / p}$ and use the substitution $w=z^{p}$ to reduce to the case $p=1$. (Note that $\lambda_{n}^{p} \rightarrow 1$ along the $p \eta$-horocycle.)

Commuting maps. The map $S$ above belongs to the geometric limit of the semigroup $\left\langle T_{n}\right\rangle$ generated by $T_{n}$. Note that $S$ and $T$ commute; indeed when $p=1, S$ and $T$ generate a rank 2 parabolic Kleinian group.

For general commuting univalent maps $f, g \in \mathcal{G}$, define the critical exponents

$$
\begin{aligned}
\delta(f, g, x) & =\inf _{x}\left\{\delta \geq 0: \sum_{i, j}\left|\left(f^{i} g^{j}\right)^{\prime}(x)\right|_{\sigma}^{\delta}<\infty\right\}, \quad \text { and } \\
\delta(f, g) & =\inf _{x} \delta(f, g, x) .
\end{aligned}
$$

The sum above extends over all $(i, j) \in \mathbb{Z}^{2}$ such that $f^{i} g^{j}$ is defined.

Proposition 12.3. The critical exponent satisfies $\delta(S, T)=2 p /(p+1)$.

Proof. We assume $U(S)=U(T)=\{z:|z|>R\}$ for some large $R$. First suppose 
$p=1 ;$ then $\delta(S, T, \infty)=\infty$, while for $x \neq \infty$ we have

$$
\sum\left|\left(S^{i} T^{j}\right)^{\prime}(x)\right|_{\sigma}^{\delta} \asymp \sum \frac{1}{\left(1+\left|\left(S^{i} T^{j}\right)(x)\right|^{2}\right)^{\delta}} \asymp \sum \frac{1}{\left(1+|i+j \tau|^{2}\right)^{\delta}} .
$$

Since $\left(S^{i} T^{j}\right)(x)$ is defined at least for all $(i, j)$ in a half-plane in $\mathbb{Z}^{2}$, we have $\delta(S, T, x)=1$. For $p>1$, make the substitution $w=z^{p}$; then $\sigma(w)|d w| \asymp$ $|d w| /|w|^{1+1 / p}$ for large $w$, so

$$
\sum\left|\left(S^{i} T^{j}\right)^{\prime}(x)\right|_{\sigma}^{\delta} \asymp \sum\left(\frac{1}{|i+j \tau|}\right)^{(1+1 / p) \delta}
$$

and therefore $\delta(S, T)=2 p /(p+1)$.

Proof of Theorem 12.1. (Dimension along horocycles). Let $\delta=\liminf \alpha\left(f_{n}\right)$, and choose normalized invariant densities $\mu_{n}$ for $f_{n}$ of dimension $\alpha\left(f_{n}\right)$. Passing to a subsequence, we can assume $\alpha\left(f_{n}\right) \rightarrow \delta$ and $\mu_{n}$ converges to an $f$-invariant density $\mu$ of dimension $\delta$.

Next we conjugate $f_{n} \rightarrow f$ to the model mappings $T_{n} \rightarrow T$ of (12.2). To do this, observe that the proof of Theorem 8.3 applies even when $\lambda_{n} \rightarrow 1$ along the $\eta$-horocycle, so long as $\eta$ is sufficiently small. Indeed, the key point of the proof is prevent recurrence of orbits of the renormalized mappings $R F_{n}$, and for this we only need $\log \left|R\left(\lambda_{n}\right)\right|$ large. Since $\log \left|R\left(\lambda_{n}\right)\right| \sim 4 \pi^{2} / \eta$ by (8.4), we obtain the following statement:

After passing to a subsequence, there is a $(1+\epsilon)$-quasiconformal change of coordinates $\phi_{n} \rightarrow \phi$, defined near $c$ and sending $\left(f_{n}, c_{n}\right) \rightarrow$ $(f, c)$ to $\left(T_{n}, \infty\right) \rightarrow(T, \infty)$. Here $\epsilon=\epsilon(\eta, f) \rightarrow 0$ as $\eta \rightarrow 0$.

After passing to a further subsequence, we can assume $T_{n}^{k(n)} \rightarrow S$ as above. It follows that $f_{n}^{k(n)}(z)$ converges, on a neighborhood of $c$, to a holomorphic map $g(z)=\left(\phi^{-1} S \phi\right)(z)$. Since $\mu_{n}$ is $f_{n}$-invariant, the density $\mu$ is also $g$-invariant.

Because $c \in J(f)$, we can find $x$ arbitrarily close to $c$ in the support of $\mu$. Near $c$, the maps $(f, g)$ behave like $(S, T)$, i.e. like the $p$-fold cover of a pair of independent translations. Thus any $x$ close enough to $c$ is contained in a ball $B$ such that for $|i|,|j| \gg 0$, the images $f^{i} g^{j}(B)$ are disjoint, and $f^{i} g^{j} \mid B$ is univalent with bounded distortion. Therefore we have

$$
\sum\left(\operatorname{diam}\left(f^{i} g^{j}\right)(B)\right)^{\delta} \asymp \sum \mu\left(f^{i} g^{j}\right)(B)=\mu\left(\bigcup\left(f^{i} g^{j}\right)(B)\right) \leq 1 .
$$

The sums extends over $(i, j)$ such that $\left(f^{i} g^{j}\right)(B)$ is defined and near $c$. 
On the other hand, setting $y=\phi(x)$ and $A=\phi(B)$, we have

$$
\begin{aligned}
\sum\left|\left(S^{i} T^{j}\right)^{\prime}(y)\right|^{\delta(1+\epsilon)} & \asymp \sum\left(\operatorname{diam}\left(S^{i} T^{j}\right)(A)\right)^{\delta(1+\epsilon)} \\
& =O\left(\sum\left(\operatorname{diam}\left(f^{i} g^{j}\right)(B)\right)^{\delta}\right),
\end{aligned}
$$

by $(1+\epsilon)$-Hölder continuity of $\phi^{-1}$. Therefore $\delta(1+\epsilon) \geq \delta(S, T)=2 p /(p+1)$. It follows that

$$
\lim \inf \alpha\left(f_{n}\right)=\delta \geq \frac{2 p}{p+1}-2 \epsilon,
$$

and since $\epsilon(\eta, f) \rightarrow 0$ as $\eta \rightarrow 0$, the proof is complete.

Remarks. The proof above emphasizes the reduction to Kleinian groups. A direct analysis along the lines of Proposition 3.2 would show $\delta(f, g)=2 p /(p+1)$, and thus in Theorem 12.1 we can actually take $\epsilon=0$ for $\eta$ sufficiently small.

Related lower bounds on H. $\operatorname{dim} J(f)$, using Ecalle cylinders, appear in [38]; see also [43], [49].

\section{Quadratic polynomials}

In this section we illustrate our main results in the setting of quadratic polynomials. Let

$$
f(z)=\lambda z+z^{2}
$$

where $\lambda$ is a primitive $p$ th root of unity. The parabolic point $z=0$ attracts the critical point $z=-\lambda / 2$ of $f$, so $f$ is geometrically finite. All periodic points of $f$ in $\mathbb{C}$ other than $z=0$ are repelling.

We claim $(f, 0)$ has $p$ petals. Indeed, every petal must contain a critical value of $f^{p}\left[7\right.$, Thm. 2.3], and $f^{p}$ has only $p$ critical values in $\mathbb{C}$.

Now let

$$
f_{n}(z)=\lambda_{n} z+z^{2}
$$

where $\lambda_{n} \rightarrow \lambda$. Let us say $\lambda_{n} \rightarrow \lambda$ radially if $\lambda_{n}^{p} \rightarrow 1$ radially; equivalent, if $\lambda_{n} / \lambda \rightarrow 1$ radially. We adopt a similar convention for horocyclic convergence.

Theorem 13.1. If $\lambda$ is a primitive pth root of unity, and $\lambda_{n} \rightarrow \lambda$ radially, then $J\left(f_{n}\right) \rightarrow J(f)$, H. $\operatorname{dim} J\left(f_{n}\right) \rightarrow$ H. $\operatorname{dim} J(f)$, and the canonical densities satisfy $\mu_{n} \rightarrow \mu$ in the weak topology on measures. 
Proof. Since $f$ has $p$ petals at $z=0,\left(f_{n}, 0\right) \rightarrow(f, 0)$ dominantly by Proposition 7.3, and the result follows from Theorem 11.2 on dynamic convergence.

Theorem 13.2. If $\lambda$ is a primitive pth root of unity, then there exist $\left|\lambda_{n}\right|<1$ with $\lambda_{n} \rightarrow \lambda$ horocyclically, such that

$$
\liminf \text { H. } \operatorname{dim} J\left(f_{n}\right) \geq \frac{2 p}{p+1} .
$$

Proof. By Theorem 12.1, for $\eta=-1 / n$ there exist $\lambda_{n}$ on the $\eta$-horocycle with $\left|\lambda_{n}-1\right|<1 / n$ and H. $\operatorname{dim} J\left(f_{n}\right)>2 p /(p+1)-\epsilon_{n}$, where $\epsilon_{n} \rightarrow 0$. Since $\eta<0$ we have $\left|\lambda_{n}\right|<1$.

Corollary 13.3. There exist $\left|\lambda_{n}\right|<1$ such that

$$
\text { H. } \operatorname{dim} J\left(\lambda_{n} z+z^{2}\right) \rightarrow 2 \text {. }
$$

Real quadratics. Finally consider the family of real quadratic polynomials

$$
f_{c}(z)=z^{2}+c
$$

Let $c_{\text {Feig }}=-1.401155 \ldots$ denote the Feigenbaum point, i.e. the limit of the cascade of period doublings as $c$ decreases along the real axis.

Theorem 13.4. The function H. $\operatorname{dim} J\left(f_{c}\right)$ is continuous for $c \in\left(c_{\text {Feig }}, 1 / 4\right]$.

Proof. For $c \in\left(c_{\text {Feig }}, 1 / 4\right)$ it is known that $f_{c}$ has either an attracting cycle of period $2^{n}$, or a parabolic point $p$ of period $2^{n}$ with two petals and multiplier -1 (see, e.g. [17]). In the attracting case, $f_{c}$ is expanding and continuity of dimension is immediate (11.1). In the parabolic case, since the multiplier $\lambda=-1$, the point $(p, p)$ is a transverse intersection of the diagonal $y=x$ with the graph of the equation $y=f_{c}^{2^{n}}(x)$ in $\mathbb{R} \times \mathbb{R}$. By transversality, if $c_{n} \rightarrow c$ in $\mathbb{R}$, then there are periodic points $p_{n}$ for $f_{n}(z)=z^{2}+c_{n}$ with $p_{n} \rightarrow p$ in $\mathbb{R}$, and with multipliers

$$
\lambda_{n}=\left(f^{2^{n}}\right)^{\prime}\left(p_{n}\right) \rightarrow-1
$$

along the real axis. Thus $f_{c_{n}} \rightarrow f_{c}$ radially, so H. $\operatorname{dim} J\left(f_{c_{n}}\right) \rightarrow$ H. $\operatorname{dim} J\left(f_{c}\right)$ by Theorem 11.2.

Finally for $c=1 / 4, p=1 / 2$ is a parabolic fixed-point of $f_{c}$ with one petal and multiplier $\lambda=1$. If $c_{n} \rightarrow c=1 / 4$ from below, then there are real fixed-points $p_{n} \rightarrow 1 / 2$ with real multipliers $\lambda_{n}=1-\sqrt{1-4 c_{n}}$, so $\lambda_{n} \rightarrow \lambda$ radially and the 
dimension is continuous in this case as well.

\section{Notes.}

1. Shishikura has shown there exist (geometrically infinite) quadratic polynomials with H. $\operatorname{dim} J(f)=2$ [38]. His argument shows directly that hyp-dim $(f)=2$, and from this he deduces that the Mandelbrot set $M$ satisfies H. $\operatorname{dim} \partial M=2$.

2. Theorems 13.1 and 13.2 generalize to $f_{n}$ in any hyperbolic component of the Mandelbrot set, with $\lambda_{n}$ denoting the multiplier of the attracting cycle. Similar results hold in the family $f_{c}(z)=z^{d}+c, d>1$.

3. As $c \rightarrow 1 / 4$ from above, the fixed-points of $f_{c}(z)=z^{2}+c$ are repelling and their multipliers tend to 1 along a horocycle. Douady, Sentenac and Zinsmeister have shown that H. $\operatorname{dim} J\left(f_{c}\right)$ is discontinuous as $c \rightarrow 1 / 4$ from above [16]. The Julia set varies discontinuously as well [13, Thm. 11.3].

Another example of discontinuity in the quadratic family is given in the next section.

4. Continuity of H. $\operatorname{dim} J\left(f_{c}\right)$ as $c \rightarrow 1 / 4$ from below was also shown in [5].

Addendum, February 1998: Zinsmeister has recently given a simplified proof that H. $\operatorname{dim}(\partial M)=2$, based ideas similar to those we present above [49].

\section{Examples of discontinuity}

We conclude with two examples of rational maps such that

(a) $f_{n} \rightarrow f$ algebraically, and

(b) $f$ and $f_{n}$ are geometrically finite, but

(c) H. $\operatorname{dim} J\left(f_{n}\right)$ does not tend to H. $\operatorname{dim} J(f)$, even though

(d) $J\left(f_{n}\right) \rightarrow J(f)$ in the Hausdorff topology.

These examples show the necessity of the assumptions in Theorem 11.2 on convergence of dimension.

I. Critical relations. The first example is a sequence of quadratic polynomials such that $f_{n} \rightarrow f$ radially but

$$
\text { H. } \operatorname{dim} J\left(f_{n}\right) \rightarrow 2>\text { H. } \operatorname{dim} J(f) .
$$

This example shows the continuity of Hausdorff dimension fails if we drop the assumption that critical relations are preserved in Theorem 11.2(a).

Let $f(z)=z^{2}+c$ where $c$ is a Misiurewicz point; that is, suppose the critical point $z=0$ is strictly preperiodic. Then the Julia set is a dendrite with H. $\operatorname{dim} J(f)<2$ by Corollary 6.2. (For a concrete example one can take $c=-2$, 
with $J(f)=[-2,2]$.

Choose expanding maps $g_{n}(z)=z^{2}+a_{n}$ with connected Julia sets such that H. $\operatorname{dim} J\left(g_{n}\right) \rightarrow 2$ (using, e.g. Corollary 13.3). In [29, Thm. 1.3] we show one can choose $c_{n} \rightarrow c$ such that $f_{n}(z)=z^{2}+c_{n}$ is renormalizable, with a suitable restriction

$$
f_{n}^{k(n)}: U_{n} \rightarrow V_{n}
$$

quadratic-like and $\left(1+\epsilon_{n}\right)$-quasiconformally conjugate to $g_{n}$, where $\epsilon_{n} \rightarrow 0$. Since $J\left(f_{n}\right)$ contains a nearly conformal copy of $J\left(g_{n}\right)$, we have H. $\operatorname{dim} J\left(f_{n}\right) \rightarrow 2$.

Since $g_{n}$ is expanding, $f_{n}$ is expanding, and $f_{n} \rightarrow f$ radially because $f$ has no parabolic points. Finally $J\left(f_{n}\right) \rightarrow J(f)$ because the Julia set $J\left(z^{2}+c\right)$ varies continuously at each Misiurewicz point $c$ [13, Cor. 5.2].

In this example, any weak limit $\mu$ of the canonical invariant densities $\mu_{n}$ on $J\left(f_{n}\right)$ must be an atomic measure living on the inverse orbit of the critical point $z=0$ (Corollary 6.6). The atom of $\mu$ at $z=0$ is the limit of the Hausdorff measures on small, renormalized copies of $J\left(g_{n}\right)$ in $J\left(f_{n}\right)$.

II. Horocyclic convergence. The second example is a sequence of quadratic rational maps such that $f_{n} \rightarrow f$ horocyclically, preserving critical relations, but

$$
\lim \text { H. } \operatorname{dim} J\left(f_{n}\right)=1>\text { H. } \operatorname{dim} J(f)=1 / 2+\epsilon .
$$

This example shows continuity of the Hausdorff dimension fails if we drop the condition H. $\operatorname{dim} J(f)>2 p /(p+1)$ in Theorem 11.2(b). We obtain such an example for any $\epsilon$ with $0<\epsilon<1 / 2$.

For $r>0$, let

$$
f(z)=z+1-\frac{r}{z}
$$

The map $f$ is geometrically finite, $c=\infty$ is a parabolic fixed-point with one petal, and $J(f) \subset \widehat{\mathbb{R}}=\mathbb{R} \cup\{\infty\}$ is a Cantor set with H. $\operatorname{dim} J(f)<1$. (Note that $f^{-1}(\widehat{\mathbb{R}})=\widehat{\mathbb{R}}$, so Corollary 6.3 applies.)

As $r$ varies from 0 to $\infty, H . \operatorname{dim} J(f)$ varies continuous from $1 / 2$ to 1 ; in fact for $r$ small,

$$
\text { H. } \operatorname{dim} J(f)=\frac{1+\sqrt{r}}{2}+O(r)
$$

(see the discussion of quadratic Blaschke products in [26]). So for $0<\epsilon<1 / 2$ we can choose $r$ such that H. $\operatorname{dim} J(f)=1 / 2+\epsilon$. 
We claim there is a sequence $\lambda_{n} \rightarrow 1$ horocyclically such that (14.1) holds for

$$
f_{n}(z)=\lambda_{n} z+1-\frac{r}{z}
$$

This is immediate from Theorem 12.1. Indeed, for each $\eta=1 / n$ there is a point $\lambda_{n}$ on the $\eta$-horocycle such that H. $\operatorname{dim} J\left(f_{n}\right) \geq 1-\epsilon(\eta, f)$ and $\left|\lambda_{n}-1\right|<1 / n$. Since $\epsilon(\eta, f) \rightarrow 0$ as $\eta \rightarrow 0$, we have $\liminf \mathrm{H}$. $\operatorname{dim} J\left(f_{n}\right) \geq 1$. But if $\lim \sup \mathrm{H}$. $\operatorname{dim} J\left(f_{n}\right)$ were to exceed 1 , we would have $\mathrm{H} . \operatorname{dim} J(f)>1$ by Theorem 11.2, which is impossible since $J(f) \subset \widehat{\mathbb{R}}$. Thus we deduce (14.1). Note that $f_{n} \rightarrow f$ dominantly since $f$ has only one petal.

Since the critical points $z= \pm \sqrt{-r}$ for $f$ are both attracted to $z=\infty$, the sequence $f_{n} \rightarrow f$ vacuously preserves critical point relations. By Theorem 9.1, $J\left(f_{n}\right) \rightarrow J(f)$, and $f_{n}$ is geometrically finite (in fact expanding) for $n \gg 0$.

In this example, any weak limit $\mu$ of the canonical invariant densities $\mu_{n}$ on $J\left(f_{n}\right)$ must be an atomic measure living on the inverse orbit of $z=\infty$ under $f$. The Hausdorff measure on $J\left(f_{n}\right)$ concentrates on small spiral arms near the parabolic point $z=\infty$ and its inverse images.

A similar example in the setting of Kleinian groups is presented in [28, §8]. There we construct geometrically finite groups with $\Gamma_{n} \rightarrow \Gamma$ strongly, such that the limit sets converge in the Hausdorff topology but

$$
\text { H. } \operatorname{dim} \Lambda\left(\Gamma_{n}\right) \rightarrow 1>\text { H. } \operatorname{dim} \Lambda(\Gamma)=1 / 2+\epsilon .
$$

The group $\Gamma$ is Fuchsian and its limit set $\Lambda(\Gamma)$, like $J(f)$ above, is a Cantor set lying on a circle.

\section{Acknowledgements}

I would like to thank A. Douady, J. Graczyk, T. Kawahira, F. Przytycki, T. Sugawa, M. Urbański and the referee for helpful correspondence.

\section{References}

[1] J. Aaronson, M. Denker, and M. Urbański, Ergodic theory for Markov fibered systems and parabolic rational maps. Trans. Amer. Math. Soc. 337 (1993), 495-548.

[2] L. Ahlfors, Lectures on Quasiconformal Mappings. Van Nostrand, 1966.

[3] A. Beardon, Iteration of Rational Functions. Springer-Verlag, 1991.

[4] C. J. Bishop and P. W. Jones, Hausdorff dimension and Kleinian groups. Acta Math. 179 (1997), 1-39.

[5] O. Bodart and M. Zinsmeister, Quelques résultats sur la dimension de Hausdorff des ensembles de Julia des polynômes quadratiques. Fund. Math. 151 (1996), 121-137.

[6] C. Camacho, On the local structure of conformal mappings and holomorphic vector fields in $\mathbf{C}^{2}$. In: Journées Singulières de Dijon, pages 83-94. Astérisque, volume 59-60, 1978. 
[7] L. Carleson and T. Gamelin, Complex Dynamics. Springer-Verlag, 1993.

[8] L. Carleson, P. W. Jones, and J.-C. Yoccoz, Julia and John. Bol. Soc. Brasil. Mat. 25 (1994), 1-30.

[9] G. Cui, Geometrically finite rational maps with given combinatorics. Preprint, 1997.

[10] M. Denker, D. Mauldin, Z. Nitecki, and M. Urbański, Conformal measure for rational functions revisited. Fund. Math. 157 (1998), 161-173.

[11] M. Denker and M. Urbański, On Sullivan's conformal measures for rational maps of the Riemann sphere. Nonlinearity 4 (1991), 365-384.

[12] M. Denker and M. Urbański, Geometric measures for parabolic rational maps. Ergod. Th. E Dynam. Sys. 12 (1992), 53-66.

[13] A. Douady, Does a Julia set depend continuously on the polynomial? In: R. Devaney (ed.), Complex Analytic Dynamics. AMS Proc. Symp. Appl. Math. 491994.

[14] A. Douady and J. Hubbard, Étude dynamique des polynômes complexes. Pub. Math. d'Orsay 84-2, 85-4, 1985.

[15] A. Douady and J. Hubbard, A proof of Thurston's topological characterization of rational maps. Acta Math. 171 (1993), 263-297.

[16] A. Douady, P. Sentenac, and M. Zinsmeister, Implosion parabolique et dimension de Hausdorff. C. R. Acad. Sci. Paris Sér. I Math. 325 (1997), 765-772.

[17] M. Feigenbaum, Universal behavior in nonlinear systems. In: P. Cvitanović (ed.), Universality in Chaos, pages 49-84. Adam Hilger Ltd, 1984.

[18] F. Gardiner, Teichmüller Theory and Quadratic Differentials. Wiley Interscience, 1987.

[19] J. Graczyk and S. Smirnov, Collet, Eckmann, \& Hölder. Invent. Math. 133 (1998), 69-96.

[20] J. Graczyk and S. Smirnov, The Fibonacci Julia set, conformal measures and Hausdorff dimension. Preprint.

[21] J. G. Hocking and G. S. Young, Topology. Addison-Wesley, 1961.

[22] M. Lyubich, On the Lebesgue measure of the Julia set of a quadratic polynomial. Stony Brook IMS Preprint 1991/10.

[23] M. Lyubich and Y. Minsky, Laminations in holomorphic dynamics. J. Diff. Geom. 47 (1997), 17-94.

[24] C. McMullen, Complex Dynamics and Renormalization, volume 135 of Annals of Math. Studies. Princeton University Press, 1994.

[25] C. McMullen, The classification of conformal dynamical systems. In Current Developments in Mathematics, 1995, pages 323-360. International Press, 1995.

[26] C. McMullen, Hausdorff dimension and conformal dynamics III: Computation of dimension. Amer. J. Math. 120 (1998), 691-721.

[27] C. McMullen, Self-similarity of Siegel disks and the Hausdorff dimension of Julia sets. Acta Math. 180 (1998), 247-292.

[28] C. McMullen, Hausdorff dimension and conformal dynamics I: Kleinian groups and strong limits. J. Diff. Geom. 51 (1999), 471-515.

[29] C. McMullen, The Mandelbrot set is universal. In: Tan Lei, ed. The Mandelbrot Set, Theme and Variations. pp. 1-18. Cambridge University Press, 2000.

[30] J. Milnor, Dynamics in One Complex Variable: Introductory Lectures. Vieweg, 1999.

[31] S. J. Patterson, The limit set of a Fuchsian group. Acta Math. 136 (1976), 241-273.

[32] F. Przytycki, Lyapunov characteristic exponents are nonnegative. Proc. Amer. Math. Soc. 119 (1993), 309-317.

[33] F. Przytycki, On measure and Hausdorff dimension of Julia sets of holomorphic ColletEckmann maps. In: International Conference on Dynamical Systems (Montevideo, 1995), volume 362 of Pitman Res. Notes Math Ser., pages 167-181. Longman, 1996.

[34] F. Przytycki, Iterations of holomorphic Collet-Eckmann maps: conformal and invariant measures. Trans. Amer. Math. Soc. 350 (1998), 717-742.

[35] F. Przytycki, Conical limit set and Poincaré exponent for iterations of rational functions. Trans. Amer. Math. Soc. 351 (1999), 2081-2099. 
[36] F. Przytycki and M. Urbański, Fractals in the Plane - Ergodic Theory Methods. Cambridge University Press, to appear.

[37] M. Shishikura, The boundary of the Mandelbrot set has Hausdorff dimension two. In Complex Analytic Methods in Dynamical Systems (Rio de Janeiro, 1992), pages 389-406. Astérisque, volume 222, 1994.

[38] M. Shishikura, The Hausdorff dimension of the boundary of the Mandelbrot set and Julia sets. Annals of Math. 147 (1998), 225-267.

[39] E. M. Stein, Harmonic Analysis. Princeton Univeristy Press, 1993.

[40] N. Steinmetz, Rational Iteration. de Gruyter Studies in Mathematics 16, 1993.

[41] D. Sullivan, Conformal dynamical systems. In Geometric Dynamics, volume 1007 of Lecture Notes in Mathematics, pages 725-752. Springer-Verlag, 1983.

[42] D. Sullivan, Entropy, Hausdorff measures new and old and limit sets of geometrically finite Kleinian groups. Acta Math. 153 (1984), 259-277.

[43] Tan L., Hausdorff dimension of subsets of the parameter space for families of rational maps. Nonlinearity 11 (1998), 233-246.

[44] W. P. Thurston, On the combinatorics and dynamics of iterated rational maps. Preprint.

[45] M. Urbański, Rational functions with no recurrent critical points. Ergod. Th. E Dynam. Sys. 14 (1994), 391-414.

[46] M. Urbański, On some aspects of fractal dimensions in higher dimensional dynamics. In: Proceedings of Workshop: Problems in higher dimensional dynamics, pages 18-25. Preprint SFB 170 Göttingen, 1995.

[47] S. M. Voronin, Analytic classification of germs of conformal mappings $(\mathbf{C}, 0) \rightarrow(\mathbf{C}, 0)$ with identity linear part. Functional Analysis Appl. 15 (1981), 1-13.

[48] J.-C. Yoccoz, Petits diviseurs en dimension 1. Astérisque, volume 231, 1995.

[49] M. Zinsmeister, Fleur de Leau-Fatou et dimension de Hausdorff. C. R. Acad. Sci. Paris Sér. I Math. 326 (1998), 1227-1232.

Curtis T. McMullen

Mathematics Department

Harvard University

1 Oxford Street

Cambridge, MA 02138-2901

USA

(Received: April 7, 1998) 